\title{
Chemistry as a probe of the structures and evolution of massive star-forming regions
}

\author{
S. D. Doty ${ }^{1}$, E. F. van Dishoeck ${ }^{2}$, F. F. S. van der $\operatorname{Tak}^{2,3}$, and A. M. S. Boonman ${ }^{2}$ \\ 1 Department of Physics and Astronomy, Denison University, Granville, OH 43023, USA \\ 2 Sterrewacht Leiden, PO Box 9513, 2300 RA Leiden, The Netherlands \\ 3 Max-Planck-Institut für Radioastronomie, Auf dem Hügel 69, 53121 Bonn, Germany
}

Received 31 October 2001 / Accepted 15 April 2002

\begin{abstract}
We present detailed thermal and gas-phase chemical models for the envelope of the massive star-forming region AFGL 2591. By considering both time- and space-dependent chemistry, these models are used to study both the physical structure proposed by van der Tak et al. $(1999,2000)$, as well as the chemical evolution of this region. The model predictions are compared with observed abundances and column densities for 29 species. The observational data cover a wide range of physical conditions within the source, but significantly probe the inner regions where interesting high-temperature chemistry may be occurring. Taking appropriate care when comparing models with both emission and absorption measurements, we find that the majority of the chemical structure can be well-explained. In particular, we find that the nitrogen and hydrocarbon chemistry can be significantly affected by temperature, with the possibility of high-temperature pathways to HCN. While we cannot determine the sulphur reservoir, the observations can be explained by models with the majority of the sulphur in CS in the cold gas, $\mathrm{SO}_{2}$ in the warm gas, and atomic sulphur in the warmest gas. Because the model overpredicts $\mathrm{CO}_{2}$ by a factor of 40, various high-temperature destruction mechanisms are explored, including impulsive heating events. The observed abundances of ions such as $\mathrm{HCO}^{+}$and $\mathrm{N}_{2} \mathrm{H}^{+}$and the cold gas-phase production of $\mathrm{HCN}$ constrain the cosmic-ray ionization rate to $\sim 5.6 \times 10^{-17} \mathrm{~s}^{-1}$, to within a factor of three. Finally, we find that the model and observations can simultaneously agree at a reasonable level and often to within a factor of three for $7 \times 10^{3} \leq t(\mathrm{yrs}) \leq 5 \times 10^{4}$, with a strong preference for $t \sim 3 \times 10^{4}$ yrs since the collapse and formation of the central luminosity source.
\end{abstract}

Key words. stars: formation - stars: individual: AFGL 2591 - ISM: molecules

\section{Introduction}

The distribution and composition of dust and gas around isolated low-mass young stellar objects (YSOs) has been well-studied both observationally and theoretically. Unfortunately, much less is known about the distribution and composition of material around high-mass YSOs (see e.g., Churchwell 1993, 1999). The higher densities and masses, and shorter lifetimes associated with massive star formation suggest that differences between regions of highand low-mass star formation can be expected.

Recent observational advances (e.g., submillimeter beams of $\sim 15^{\prime \prime}$ sampling smaller regions of higher critical densities, interferometry at 1 and $3 \mathrm{~mm}$, and ground- and space-based infrared observations of gas and ices) have led to a new and better understanding of the environment around massive YSOs (see e.g., Garay \& Lizano 1999; van Dishoeck \& Hogerheijde 1999; Hatchell et al. 2000;

Send offprint requests to: S. Doty,

e-mail: doty@cc.dension.edu
Beuther et al. 2002). In this vein, van der Tak et al. (1999, 2000) have conducted detailed multi-wavelength studies of high-mass YSOs, and begun to form a picture for the physical structure of some of these regions. The proposed material distributions in the envelopes fit a wide variety of continuum and spectral line data. However, they are incomplete without a detailed thermal and chemical structure. The proposed material distribution can be used to test the chemical structure and evolution of the envelope, and the combined results can eventually be used to compute line strengths and profiles for direct comparison with observations.

Significant work has been involved in developing an understanding of the chemistry of star-forming regions. This ranges from studies of cold, dark clouds (e.g., Herbst \& Klemperer 1973; Prasad \& Huntress 1980; Leung et al. 1984; Gwenlan et al. 2000) to "hot cores" (see e.g., reviews by Millar 1993; Walmsley \& Schilke 1992; Kurtz et al. 2000). In nearly all cases, however, the chemistry is considered for a homogeneous cloud, or a point within a 
cloud (see, though, Xie et al. 1995 and Bergin et al. 1995 for counter examples). Unfortunately, the physical conditions (i.e., temperature and density) vary strongly with position within the envelope, meaning that potentially extreme chemical variations may occur between the source center and the observer. It is this strong variation of chemical composition with position and time that may provide one of the best benchmarks of our understanding of both the structures and evolution of massive star-forming regions.

In this paper, we utilize position-dependent thermal balance and time- and position-dependent chemical modeling to probe the validity of the physical structures proposed by van der Tak et al. (1999 \& 2000), and more importantly, to study the chemical evolution of AFGL 2591. In particular, taking their structure as a starting point, we construct detailed models for the gas-phase chemistry of this source, and compare the results with observations. AFGL 2591 is a massive $\left(\sim 42 M_{\odot}\right.$ within $\left.r=3 \times 10^{4} \mathrm{AU}\right)$, luminous $\left(\sim 2 \times 10^{4} L_{\odot}\right)$ infrared source with many of the properties thought to characterize YSOs. While most massive stars form in clusters, AFGL 2591 has the advantage that it is forming in relative isolation - allowing us to study its physical, thermal, and chemical structures without influence from other nearby massive sources. It has the further advantage of being well-observed both in the continuum and in a variety of molecular lines.

This paper is organized as follows. The existing observations providing the model constraints are briefly discussed in Sect. 2. In Sect. 3, the model is described. The model is then applied to AFGL 2591 and compared with the observational results in Sect. 4. In Sect. 5, we compare our time-dependent model predictions with the observations in order to constrain the chemical age of the envelope. Finally, we summarize our results and conclude in Sect. 6 .

\section{Existing observations and usage}

AFGL 2591 has been well-observed both in the continuum and in various molecular lines. While no new observations are presented in this paper, it is important to briefly note and discuss the relevant observations as they provide the constraints placed on the model.

\subsection{Continuum}

AFGL 2591 has been observed in the range 2-60000 $\mu \mathrm{m}$ by Lada et al. (1984), Aitken (1988), Sandell (1998, private communication), and van der Tak et al. (1999). These results were analyzed by van der Tak et al. (2000 - see Sect. 3 below) to constrain the density distribution and grain properties - necessary for not only the thermal structure, but also to properly evaluate the gas thermal balance and hence obtain the gas temperature as a function of position.

\subsection{Molecular lines}

A wide variety of observations, both in the infrared and submillimeter, have been conducted of molecular gas in AFGL 2591, some of which are as of yet unpublished. The results are summarized in Table 1, where the species, observed abundance $\left[x(\mathrm{X}) \equiv n(\mathrm{X}) / n\left(\mathrm{H}_{2}\right)\right]$ or column density $[N(\mathrm{X})]$, inferred excitation temperature, method of analysis, weight used in selecting the most important of the relevant observations, type of observation, and reference are listed.

\subsection{Notes on Table 1 and usage of the data}

The observation type is listed in Table 1 as this is significant for comparing the results with observations. For infrared absorption lines, the molecules observed are along the (narrow) line of sight to the background continuum source. Consequently, these results should be compared to model "radial column densities", namely $N_{\text {radial }} \equiv \int n(r) \mathrm{d} r$. On the other hand, submillimeter emission lines arise from throughout the envelope. In these cases, averages over the beam are used in comparing predicted and observed column densities. Here, the "beam-averaged column density" is defined as $N_{\text {beam }} \equiv$ $\iint n(z, p) \mathrm{d} z G(p) 2 \pi p \mathrm{~d} p / \int G(p) 2 \pi p \mathrm{~d} p$ where $p$ is the impact parameter, and $G(p)$ is the beam response function. We also divide the data in this fashion, as we expect many of the uncertainties in the analysis to be similar for one type of observation.

In Cols. 2 and 3 of Table 1 we list the inferred fractional abundance or column density of the given molecule toward AFGL 2591. This is done to provide the most comprehensive set of information with which to compare our models.

While determination of column density is relatively straightforward for infrared absorption lines in the limit of no re-emission, the situation is more difficult for emission lines as the emission may arise from a range of radii, and thus a range of densities, temperatures, chemical abundances, and optical depths. To combat this, some effort has been made recently to determine the fractional abundance within the envelope through detailed, non local thermodynamic equilibrium (NLTE) radiative transfer (RT) modeling (van der Tak et al. 1999). When this is done, we view the inferred abundances as superior to pure column densities as they account for many of the potential errors in determining the column density. As examples, van der Tak et al. (2000) and Boonman et al. (2001) have used such modeling to suggest "jump" models for the chemical enhancement of species within certain regions of YSO envelopes. As a result, in Col. 5 we note the method used in determining the observational result. We also use these criteria to assign a weight (higher is better) in Col. 6 to denote which data/fits we view as superior. In cases where the fit due to radiative transfer modeling is only moderate, we give this result the same weight as the results from other methods. 
Table 1. Inferred column densities and abundances toward AFGL 2591.

\begin{tabular}{|c|c|c|c|c|c|c|c|}
\hline Molecule & $x$ & $N\left(\mathrm{~cm}^{-2}\right)$ & $T_{\mathrm{ex}}(\mathrm{K})$ & Method & Weight & Data & Ref \\
\hline $\mathrm{H}_{2}$ & & $9.6(22)$ & - & Scale $N(\mathrm{CO})$ & 2 & - & $\mathrm{a}$ \\
\hline $\mathrm{HCN}^{(\alpha 1)}$ & $\sim 1(-8)$ & & $\lesssim 230$ & NLTE RT Model & 3 & submm - JCMT & $\mathrm{b}$ \\
\hline.$^{(\alpha 2)}$ & $\sim 1(-6)$ & & $\gtrsim 230$ & NLTE RT Model & 3 & submm - JCMT & $\mathrm{b}$ \\
\hline $\mathrm{HCN}^{(\alpha 1)}$ & & $4.0(16)$ & 600 & Absn. Depth & 2 & IR - ISO & $\mathrm{a}$ \\
\hline $\mathrm{HCN}^{(\beta 1)}$ & & $2.0(15)$ & $38(\mathrm{CO})$ & Absn. Depth & 2 & IR - ISO & $\mathrm{a}$ \\
\hline$\ldots{ }^{(\beta 2)}$ & & $4.5(16)$ & $1010(\mathrm{CO})$ & Absn. Depth & 2 & IR - ISO & $\mathrm{a}$ \\
\hline $\mathrm{HCN}^{(\alpha 1)}$ & & $\leq 1.7(16)$ & $38(\mathrm{CO})$ & Absn. Depth & 2 & IR - IRTF & $\mathrm{c}$ \\
\hline$\ldots{ }^{(\alpha 2)}$ & & $2.0(16)$ & $200(\mathrm{CO})$ & Absn. Depth & 2 & IR - IRTF & $\mathrm{c}$ \\
\hline.$^{(\alpha 3)}$ & & $1.6(16)$ & $1010(\mathrm{CO})$ & Absn. Depth & 2 & IR - IRTF & $\mathrm{c}$ \\
\hline $\mathrm{HNC}^{(\alpha 1)}$ & & $2.9(13)$ & - & NLTE / escape prob. & 2 & submm - JCMT & $\mathrm{d}$ \\
\hline $\mathrm{HNC}^{(\alpha 1)}$ & $1.0(-8)$ & & - & NLTE RT Model & 3 & submm - JCMT & $\mathrm{n}$ \\
\hline $\mathrm{HC}_{3} \mathrm{~N}^{(\alpha 1)}$ & & $5.0(12)$ & - & NLTE / escape prob. & 2 & submm - JCMT or CSO & $\mathrm{d}$ \\
\hline $\mathrm{HC}_{3} \mathrm{~N}^{(\alpha 1)}$ & $2.0(-8)$ & & - & NLTE RT Model & 2 & submm - JCMT & $\mathrm{n}$ \\
\hline $\mathrm{HCO}^{+(\alpha 1)}$ & $1.0(-8)$ & & - & NLTE RT Model & 3 & submm - JCMT & $\mathrm{e}$ \\
\hline $\mathrm{HCS}^{+(\alpha 1)}$ & $3.0(-10)$ & & - & NLTE RT Model & 3 & submm - JCMT & $\mathrm{n}$ \\
\hline $\mathrm{H}_{3}^{+(\alpha 1)}$ & & $2-3(14)$ & - & Absn. Depth & 2 & IR - UKIRT & $\mathrm{f}$ \\
\hline $\mathrm{H}_{2} \mathrm{O}^{(\alpha 1)}$ & & $3.5(18)$ & 450 & Absn. Depth & 2 & IR - ISO & $\mathrm{g}$ \\
\hline $\mathrm{H}_{2} \mathrm{~S}^{(\alpha 1)}$ & & $\leq 1.0(19)$ & - & Absn. Depth & 2 & IR - ISO & $\mathrm{h}$ \\
\hline $\mathrm{H}_{2} \mathrm{CO}^{(\alpha 1)}$ & $2.0(-9)$ & & - & NLTE RT Model & 3 & submm - JCMT & $\mathrm{i}$ \\
\hline $\mathrm{H}_{2} \mathrm{CO}^{(\beta 1)}$ & & $8.0(13)$ & 89 & LTE Rot. Diagram & 2 & submm - JCMT & $\mathrm{e}$ \\
\hline $\mathrm{H}_{2} \mathrm{CS}^{(\alpha 1)}$ & $1.0(-9)$ & & - & NLTE RT Model & 2 & submm - JCMT & $\mathrm{n}$ \\
\hline $\mathrm{CI}^{(\alpha 1)}$ & & $\leq 6.8(17)$ & - & NLTE / escape prob. & 2 & submm - CSO & $\mathrm{j}$ \\
\hline $\mathrm{C}^{+(\alpha 1)}$ & & $\leq 6.8(17)$ & - & LTE escape prob. & 2 & IR - ISO & $\mathrm{k}$ \\
\hline $\mathrm{C}_{2} \mathrm{H}^{(\alpha 1)}$ & $2.0(-9)$ & & - & NLTE RT Model & 3 & submm - JCMT & $\mathrm{n}$ \\
\hline $\mathrm{C}_{2} \mathrm{H}_{2}^{(\alpha 1)}$ & & $\leq 2.0(16)$ & 900 & Absn. Depth & 2 & IR - ISO & $\mathrm{a}$ \\
\hline $\mathrm{C}_{2} \mathrm{H}_{2}^{(\beta 1)}$ & & $\leq 1.0(15)$ & $38(\mathrm{CO})$ & Absn. Depth & 2 & IR - ISO & $\mathrm{a}$ \\
\hline$\ldots{ }^{(\beta 2)}$ & & $2.0(16)$ & $1010(\mathrm{CO})$ & Absn. Depth & 2 & IR - ISO & $\mathrm{a}$ \\
\hline $\mathrm{C}_{2} \mathrm{H}_{2}^{(\alpha 1)}$ & & $\leq 8.0(14)$ & $38(\mathrm{CO})$ & Absn. Depth & 2 & IR - IRTF & $\mathrm{c}$ \\
\hline.$^{(\alpha 2)}$ & & $4.2(15)$ & $200(\mathrm{CO})$ & Absn. Depth & 2 & IR - IRTF & $\mathrm{c}$ \\
\hline$\ldots{ }^{(\alpha 3)}$ & & $1.0(16)$ & $1010(\mathrm{CO})$ & Absn. Depth & 2 & IR - IRTF & $\mathrm{c}$ \\
\hline $\mathrm{CH}_{4}^{(\alpha 1)}$ & & $2.5(17)$ & $\geq 1000$ & Absn. Depth & 2 & IR - ISO & $\mathrm{h}$ \\
\hline $\mathrm{CH}_{4}^{(\alpha 1)}$ & & $\leq 8.0(15)$ & $38(\mathrm{CO})$ & Absn. Depth & 2 & IR - IRTF & c \\
\hline$\ldots{ }^{(\alpha 2)}$ & & $\leq 1.0(17)$ & $200(\mathrm{CO})$ & Absn. Depth & 2 & IR - IRTF & $\mathrm{c}$ \\
\hline$\ldots{ }^{(\alpha 3)}$ & & $\leq 1.3(18)$ & $1010(\mathrm{CO})$ & Absn. Depth & 2 & IR - IRTF & $\mathrm{c}$ \\
\hline $\mathrm{CH}_{3} \mathrm{OH}^{(\alpha 1)}$ & $2.6(-9)$ & & $\leq 90$ & NLTE RT Model & 3 & submm - JCMT & $\mathrm{i}$ \\
\hline$\ldots{ }^{(\alpha 2)}$ & $8.0(-8)$ & & $\geq 90$ & NLTE RT Model & 3 & submm - JCMT & $\mathrm{i}$ \\
\hline $\mathrm{CH}_{3} \mathrm{OH}^{(\beta 1)}$ & & $1.2(15)$ & 163 & rot. diagram & 2 & submm - JCMT & $\mathrm{i}$ \\
\hline $\mathrm{CH}_{3} \mathrm{CN}^{(\alpha 1)}$ & $2.0(-8)$ & & - & NLTE RT Model & 2 & submm - JCMT & $\mathrm{n}$ \\
\hline
\end{tabular}

In both cases, where an excitation temperature can be assigned to the data, we note the temperature for that component as $T_{\mathrm{ex}}$ in Col. 4 of Table 1 . While $T_{\mathrm{ex}}$ is not necessarily equal to the kinetic temperature, it does give some indication as to the region from which the observation arises. The values of 38,200 and $1010 \mathrm{~K}$ refer to the excitation temperatures of CO found by Mitchell et al. (1989) in infrared absorption line studies. The $200 \mathrm{~K}$ component is thought to be associated with shocked outflowing material, whereas the other two temperatures refer to the quiescent envelope.
Finally, we note the relative importance of different measurements for probing various regions in the envelope. Absorption is confined to the narrow line of sight toward the central source. For centrally-condensed envelopes, the column density is dominated by the interior. This makes absorption measurements useful for probing the warm interior. On the other hand, emission measurements can and often do arise from throughout the envelope. When the density falls off slower than $r^{-2}$ as is the case for AFGL 2591 (van der Tak et al. 1999) the outer portion of the envelope dominates the mass, and so emission 
Table 1. continued.

\begin{tabular}{|c|c|c|c|c|c|c|c|}
\hline Molecule & $x$ & $N\left(\mathrm{~cm}^{-2}\right)$ & $T_{\mathrm{ex}}(\mathrm{K})$ & Method & Weight & Data & Ref \\
\hline $\mathrm{CO}^{(\alpha 1)}$ & & $1.3(19)$ & - & Absn. Depth & 2 & IR - CFHT & o \\
\hline $\mathrm{CO}^{(\alpha 1)}$ & & $3.4(19)$ & - & NLTE RT Model & 3 & submm - JCMT & $\mathrm{e}$ \\
\hline $\mathrm{CO}_{2}^{(\alpha 1)}$ & & $2.5(16)$ & 500 & Absn. Depth & 2 & IR - ISO & $\mathrm{g}$ \\
\hline $\mathrm{CS}^{(\alpha 1)}$ & $3.0(-9)$ & & 40 & NLTE RT Model & 3 & submm - JCMT & $\mathrm{e}$ \\
\hline $\mathrm{CS}^{(\alpha 1)}$ & & $\leq 2.6(15)$ & $38(\mathrm{CO})$ & Absn. Depth & 2 & IR - IRTF & $\mathrm{c}$ \\
\hline$\ldots{ }^{(\alpha 2)}$ & & $\leq 3.4(15)$ & $200(\mathrm{CO})$ & Absn. Depth & 2 & IR - IRTF & c \\
\hline.$^{(\alpha 3)}$ & & $\leq 9.0(15)$ & $1010(\mathrm{CO})$ & Absn. Depth & 2 & IR - IRTF & $\mathrm{c}$ \\
\hline $\mathrm{CN}^{(\alpha 1)}$ & $5.0(-8)$ & & - & NLTE RT Model & 2 & submm - JCMT & $\mathrm{n}$ \\
\hline $\mathrm{OH}^{(\alpha 1)}$ & & $\geq 4.7(14)$ & - & Absn. Depth & 2 & IR - ISO & $\mathrm{h}$ \\
\hline $\mathrm{O}_{2}^{(\alpha 1)}$ & $\leq 1.0(-6)$ & & - & NLTE / opt. thin & 3 & submm - SWAS & 1 \\
\hline $\operatorname{OCS}^{(\alpha 1)}$ & & $1.0(14)$ & - & NLTE / escape prob. & 2 & submm - JCMT & $\mathrm{d}$ \\
\hline $\operatorname{OCS}^{(\alpha 1)}$ & $4.0(-8)$ & & - & NLTE RT Model & 3 & submm - JCMT & $\mathrm{n}$ \\
\hline $\mathrm{NH}_{3}^{(\alpha 1)}$ & & $\leq 5.0(14)$ & $38(\mathrm{CO})$ & Absn. Depth & 2 & IR - IRTF & $\mathrm{c}$ \\
\hline$\ldots{ }^{(\alpha 2)}$ & & $\leq 1.0(15)$ & $200(\mathrm{CO})$ & Absn. Depth & 2 & IR - IRTF & c \\
\hline$\ldots{ }^{(\alpha 3)}$ & & $\leq 7.0(15)$ & $1010(\mathrm{CO})$ & Absn. Depth & 2 & IR - IRTF & c \\
\hline $\mathrm{NH}_{3}^{(\alpha 1)}$ & $2.0(-8)$ & & - & NLTE RT Model & 2 & cm - Effelsberg & $\mathrm{n}$ \\
\hline $\mathrm{N}_{2} \mathrm{H}^{+(\alpha 1)}$ & & $1.4(12)$ & - & NLTE / escape prob. & 2 & submm - JCMT & $\mathrm{d}$ \\
\hline $\mathrm{N}_{2} \mathrm{H}^{+(\alpha 1)}$ & $5.0(-10)$ & & - & NLTE RT Model & 3 & submm - JCMT & $\mathrm{n}$ \\
\hline $\mathrm{SO}^{(\alpha 1)}$ & $2.0(-8)$ & & - & NLTE RT Model & 3 & submm - JCMT & $\mathrm{n}$ \\
\hline $\mathrm{SO}_{2}^{(\alpha 1)}$ & & $6.0(16)$ & 200 & Absn. Depth & 2 & IR - ISO & $\mathrm{m}$ \\
\hline
\end{tabular}

$a(b)$ means $a \times 10^{b}$.

In ${ }^{(\alpha 1)}$ the first symbol denotes the fit number ( $\alpha$ is the first fit, $\beta$ is the second, ...), and the second is the component of that fit ( 1 is the first component, 2 is the second, ...).

The (CO) notation signifies that $T_{\text {ex }}$ was forced to be one of the three CO temperatures from Mitchell et al. (1989).

Method \& Weight: the method used to infer, and the significance we ascribe to, the observational result (higher is better).

${ }^{\mathrm{a}}$ Lahuis \& van Dishoeck (2000), b Boonman et al. (2001), ${ }^{\mathrm{c}}$ Carr et al. (1995), ${ }^{\mathrm{d}}$ van Dishoeck (2001, private communication),

e van der Tak et al. (1999), ${ }^{\mathrm{f}}$ McCall et al. (1999), ${ }^{\mathrm{g}}$ Boonman et al. (2000), ${ }^{\mathrm{h}}$ Boonman (2001, private communication),

${ }^{\mathrm{i}}$ van der Tak et al. (2000), ${ }^{\mathrm{j}}$ Choi et al. (1994), ${ }^{\mathrm{k}}$ Wright (2001, private communication), ${ }^{1}$ Goldsmith et al. (2000), ${ }^{\mathrm{m}}$ Keane et al. (2001), ${ }^{\mathrm{n}}$ van der Tak (2002, in preparation), ${ }^{\circ}$ Mitchell et al. (1989).

measurements are often more useful for probing the cool exterior. These expectations are relatively consistent with the results of Table 1, where many of the absorption measurements include significant high excitation temperature components, while the inferred excitation temperatures for the emission data are generally much lower.

\section{Model}

In this section, a brief synopsis of the physical, thermal, and chemical models are provided. For more detailed information, see van der Tak et al. (1999, 2000), Doty \& Neufeld (1997), and references therein. For reference, the model parameters are reproduced in Table 2.

\subsection{Physical model}

Our model for AFGL 2591 concentrates on the extended envelope of source. While an inner disk may be present, OVRO interferometric observations by van der Tak et al. (1999) suggest an unresolved central source of radius $30<r(\mathrm{AU})<1000$. These and other continuum observations were analyzed by van der Tak et al. (2000) using a modified version of the self-consistent continuum radiative transfer model of Egan et al. (1988). Based upon the fit to the continuum flux and surface brightness, as well as CS line data, they constrained the density to a power law of the form $n(r)=n_{0}\left(r_{0} / r\right)^{\alpha}$. In particular, they found a best fit with $\alpha=1.0$, and $n_{0} \equiv n\left(\mathrm{H}_{2}, r=\right.$ $\left.r_{0}=2.7 \times 10^{4} \mathrm{AU}\right)=5.8 \times 10^{4} \mathrm{~cm}^{-3}$. We adopt these values for the remainder of the paper. Our inner radial position was chosen to be $r_{\text {in }}=2 \times 10^{2} \mathrm{AU}$, corresponding to $T \sim 440 \mathrm{~K}$ (see below). This inner radius was chosen not only for consistency with the observations of van der Tak et al. (2000), but also as extrapolation of a density power law further into the interior of the envelope leads to column densities inconsistent with observations (see Sect. 4.2 below).

Following the analyses of van der Tak et al. (1999, 2000) and Doty \& Neufeld (1997), we assume that the physical and thermal model does not change significantly with time so that an equilibrium may be achieved, but we do allow for a time-dependent chemical evolution. While the collapse and rise in luminosity will occur on short time scales $(<1000 \mathrm{yr})$, it is the combination of density 
Table 2. Model parameters.

\begin{tabular}{|c|c|c|}
\hline Parameter & Value & Ref. \\
\hline Outer radius $(\mathrm{AU})$ & $3.0(4)$ & $\mathrm{a}$ \\
\hline Inner radius (AU) & $2.0(2)$ & $\mathrm{a}$ \\
\hline \multicolumn{3}{|l|}{ Density $\left[n(r)=n_{0}\left(r_{0} / r\right)^{\alpha}\right]$} \\
\hline$\ldots$ Exponent $[\alpha]$ & 1.0 & $\mathrm{a}, \mathrm{b}$ \\
\hline$\ldots$ Ref. position $\left[r_{0}\right](\mathrm{AU})$ & $2.7(4)$ & $\mathrm{b}$ \\
\hline$\ldots$ Ref. $\mathrm{H}_{2}$ density $\left[n_{0}\right]\left(\mathrm{cm}^{-3}\right)$ & $5.8(4)$ & $\mathrm{b}$ \\
\hline $\mathrm{CR}$ ionization rate $[\zeta]\left(\mathrm{s}^{-1}\right)$ & $5.6(-17)$ & $\mathrm{c}$ \\
\hline \multicolumn{3}{|l|}{ Initial Abundance $(T>100 \mathrm{~K})$} \\
\hline $\mathrm{CO}$ & $3.7(-4)$ & $\mathrm{a}$ \\
\hline $\mathrm{CO}_{2}$ & $3.0(-5)$ & $\mathrm{d}$ \\
\hline $\mathrm{H}_{2} \mathrm{O}$ & $1.5(-4)$ & $\mathrm{d}$ \\
\hline $\mathrm{H}_{2} \mathrm{~S}$ & $1.6(-6)$ & see text \\
\hline $\mathrm{N}_{2}$ & $7.0(-5)$ & $\mathrm{e}$ \\
\hline $\mathrm{CH}_{4}$ & $1.0(-7)$ & e \\
\hline $\mathrm{C}_{2} \mathrm{H}_{4}$ & $8.0(-8)$ & e \\
\hline $\mathrm{C}_{2} \mathrm{H}_{6}$ & $1.0(-8)$ & $\mathrm{e}$ \\
\hline OI & $0.0(0)$ & $\mathrm{e}$ \\
\hline $\mathrm{H}_{2} \mathrm{CO}$ & $1.2(-7)$ & $\mathrm{e}$ \\
\hline $\mathrm{CH}_{3} \mathrm{OH}$ & $1.0(-6)$ & $\mathrm{e}$ \\
\hline $\mathrm{S}$ & $0.0(0)$ & e \\
\hline $\mathrm{Fe}$ & $2.0(-8)$ & e \\
\hline \multicolumn{3}{|l|}{ Initial Abundance $(T<100 \mathrm{~K})$} \\
\hline $\mathrm{CO}$ & $3.7(-4)$ & $\mathrm{a}$ \\
\hline $\mathrm{CO}_{2}$ & $0.0(0)$ & $\mathrm{f}$ \\
\hline $\mathrm{H}_{2} \mathrm{O}$ & $0.0(0)$ & $\mathrm{f}$ \\
\hline $\mathrm{H}_{2} \mathrm{~S}$ & $0.0(0)$ & $\mathrm{f}$ \\
\hline $\mathrm{N}_{2}$ & $7.0(-5)$ & $\mathrm{e}$ \\
\hline $\mathrm{CH}_{4}$ & $1.0(-7)$ & e \\
\hline $\mathrm{C}_{2} \mathrm{H}_{4}$ & $8.0(-8)$ & e \\
\hline $\mathrm{C}_{2} \mathrm{H}_{6}$ & $1.0(-8)$ & e \\
\hline OI & $8.0(-5)$ & g \\
\hline $\mathrm{H}_{2} \mathrm{CO}$ & $0.0(0)$ & $\mathrm{f}$ \\
\hline $\mathrm{CH}_{3} \mathrm{OH}$ & $0.0(0)$ & $\mathrm{f}$ \\
\hline $\mathrm{S}$ & $6.0(-9)$ & see text \\
\hline $\mathrm{Fe}$ & $2.0(-8)$ & $\mathrm{e}$ \\
\hline
\end{tabular}

$a(b)$ means $a \times 10^{b}$, All abundances are gas-phase, and relative to $\mathrm{H}_{2}$

a van der Tak et al. (1999), b van der Tak et al. (2000),

${ }^{\mathrm{c}}$ van der Tak \& van Dishoeck (2000), ${ }^{\mathrm{d}}$ Boonman et al. (2000),

e Charnley (1997), ${ }^{\mathrm{f}}$ assumed frozen-out or absent in cold gasphase, ${ }^{\mathrm{g}}$ taken to be $\sim$ consistent with Meyer et al. (1998).

structure and luminosity of the central source that sets the temperature structure. Therefore, as long as the envelope mass and luminosity do not significantly change, we can consider the source as approximately constant over the $\sim 10^{5}$ yrs in which the envelope will be dissipated (Hollenbach et al. 1994; Richling \& Yorke 1997). To see this, consider the fact that the free fall and sound-crossing times at the outer edge are both $\sim 2 \times 10^{5}$ yrs. While these timescales are smaller closer to the center, accretion events should probably only be important in the very interior.

Finally, we note that an outflow has been observed toward this source (see, e.g., Bally \& Lada 1983; Mitchell et al. 1989). However, spectroscopy shows that nearly all submillimeter lines with the exception of $\mathrm{CO}$ can be assigned to the envelope as their linewidths are only $\sim$ few $\mathrm{km} \mathrm{s}^{-1}$ (see, e.g., van der Tak et al. 1999, and recent and upcoming infrared data from TEXES by Knez et al. 2002 and Boonman et al., in preparation). Only CO has a significant fraction of the observed material in the outflow. This assignment of material to the envelope rather than the outflow is also justified a posteriori as our models are able to reproduce a good deal of the observed chemistry without the requirement of shock chemistry. Because the submillimeter lines probe high excitation gas, the lower density surrounding cloud is automatically filtered out.

\subsection{Thermal model}

The equilibrium gas temperature within the cloud is determined by the balance between heating and cooling. The gas heating is dominated by gas-grain collisions, and the dust temperature is determined from the self-consistent solution to the continuum radiative transfer problem as above. The Neufeld et al. (1995) cooling functions were adopted, with modifications as noted in Doty \& Neufeld (1997). Furthermore, as the Neufeld et al. (1995) cooling functions were constructed assuming a singular isothermal sphere (with a commensurate $n \propto r^{-2}$ density power law), they were modified to be applicable to the $r^{-1}$ power law adopted here. This entailed two corrections. First, the column densities had to be computed correctly at each position, rather than simply relying upon the local density. Second, the cooling functions for the tabular results were modified by a factor $[N(\alpha=2) / N(\alpha)]^{f}$, where $N(\alpha) \equiv \int_{r_{\text {out }}}^{r} n(r) \mathrm{d} r$ is the column density for a power law distribution $r^{-\alpha}$. Here $f$ varies linearly with $\log (N)$ from -0.5 at $N=10^{16} \mathrm{~cm}^{-2}$ to -1.0 at $N=10^{21} \mathrm{~cm}^{-2}$. We take $f=0$ for $N<10^{16} \mathrm{~cm}^{-2}$, and $f=-1.0$ for $N>10^{21} \mathrm{~cm}^{-2}$. This factor is chosen to match the functional dependence of the cooling rate on the column density as described in Neufeld et al. (1995) for $\mathrm{H}_{2} \mathrm{O}$ - the dominant coolant utilized in tabular form - and is consistent with the fact that the cooling rate should be inversely proportional to the column density for opaque sources. The resulting gas temperature distribution is shown in Fig. 1, and is physically similar to that of Doty \& Neufeld (1997), namely that $T_{\text {gas }} \sim T_{\text {dust }}$, as was assumed by van der Tak et al. (1999, 2000). For comparison, models run assuming $T_{\text {gas }}=T_{\text {dust }}$ show no significant differences

\subsection{Chemical model}

The chemical model is based upon the UMIST gas-phase chemical reaction network (Millar et al. 1997). Using this network, we construct pseudo time-dependent models of 


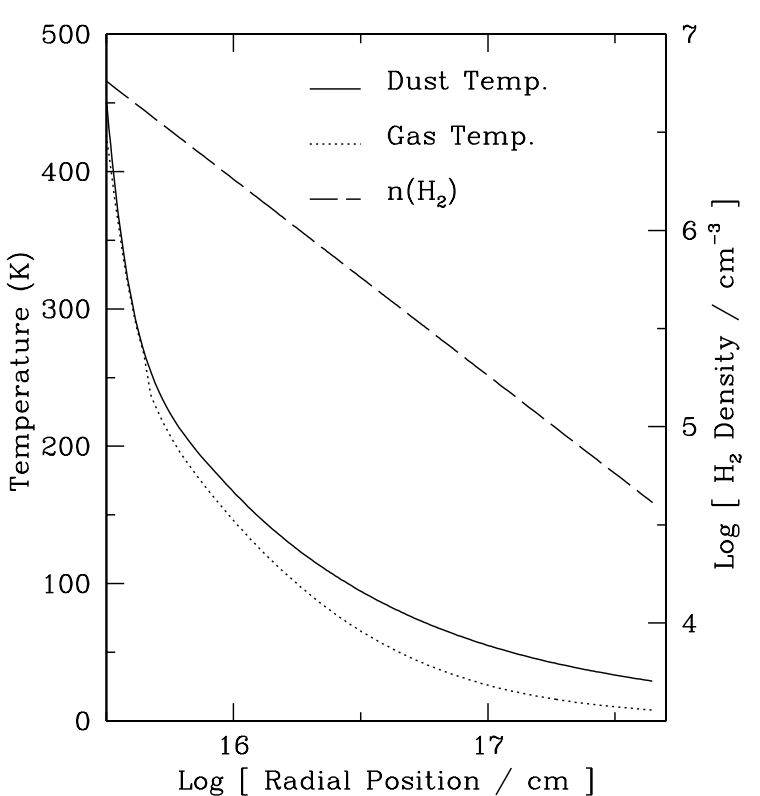

Fig. 1. Physical and thermal structure of AFGL 2591. The density and dust results from the model of van der Tak et al. (2000). The gas temperatures are calculated from the detailed thermal balance, similar to Doty \& Neufeld (1997). Note that $T_{\text {gas }} \sim T_{\text {dust }}$.

the evolution of the chemical abundances. We do this over a range of 30 radial grid points, providing a time- and space-dependent chemical evolution. The local parameters (hydrogen density, temperature, and optical depth) at each radial point are taken from the physical and thermal structure calculations above. For our initial abundances, we follow Charnley (1997; private communication). These parameters allow us to reproduce many of the results of the hot core models of Charnley (1997; private communication), with most discrepancies directly attributable to differences in adopted reaction rates.

We also include the approximate effects of freeze-out onto dust grains by initially depleting certain species below $100 \mathrm{~K}$ (see Sect. 4.7 for discussion of $\mathrm{H}_{2} \mathrm{CO}$ and $\left.\mathrm{CH}_{3} \mathrm{OH}\right)$. We attempt to minimize this effect by predominantly depleting those species that have high observed solid-phase abundances. Our initial fractional abundances relative to $\mathrm{H}_{2}$, as well as other model parameters are listed in Table 2.

The cosmic-ray ionization rate is taken from van der Tak \& van Dishoeck (2000) for AFGL 2591, and will be discussed in Sect. 4.5. The effects of cosmic-ray induced photochemistry were ignored. The initial sulphur abundance was chosen to make the models agree with observations (see Sect. 4.6). The assumed sulphur abundances are in general agreement with observations for both the warm (e.g., toward Orion by Minh et al. 1990), and the cold (e.g., Irvine et al. 1991) components.

The effects of photodissociation from the ISRF at the outer boundary are included, but are generally small due to the high optical depth, and the coarseness of the spatial grid considered.

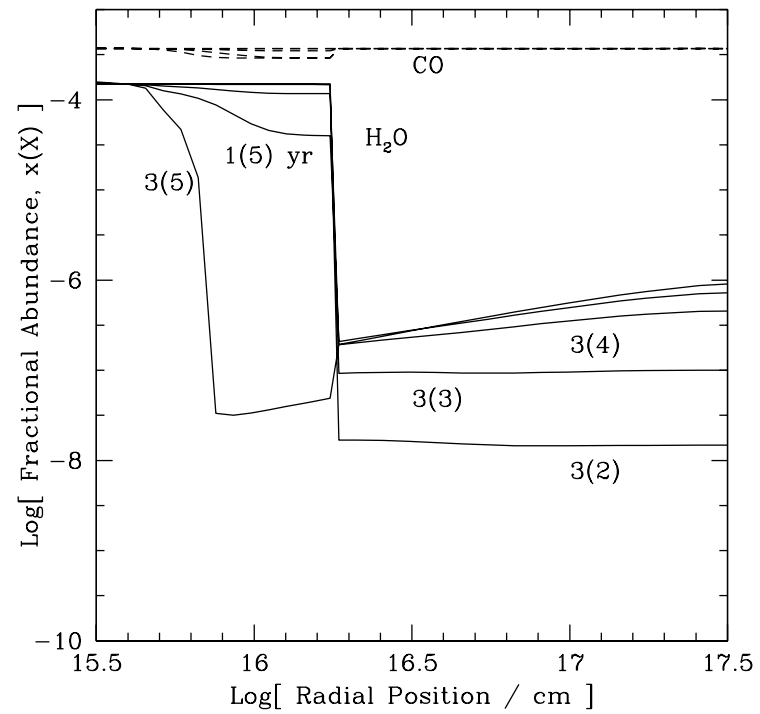

Fig. 2. The fractional abundances of $\mathrm{CO}$ and $\mathrm{H}_{2} \mathrm{O}$ throughout the envelope as a function of time. The dashed-lines correspond to the (constant) CO abundance, and the solid lines to the $\mathrm{H}_{2} \mathrm{O}$ abundance. The curves are labeled by the time in years, where $a(b)=a \times 10^{b}$.

\section{Results}

\subsection{Basic molecules: $\mathrm{H}_{2}, \mathrm{CO}$, and $\mathrm{H}_{2} \mathrm{O}$}

Due to their stability, $\mathrm{CO}$ and $\mathrm{H}_{2} \mathrm{O}$ are significant chemical sinks, with abundances that are relatively constant with time. To see this, in Fig. 2 we plot the fractional abundance of $\mathrm{CO}$ and $\mathrm{H}_{2} \mathrm{O}$ throughout the envelope as functions of time. As can be seen, the $\mathrm{CO}$ abundance is essentially constant in time. The abundance has been chosen to be consistent with observations.

The water abundance in the warm interior is nearly constant, due to the fact that the majority of the oxygen not in CO is initially placed into water. This is consistent with models we and others (e.g., Doty \& Neufeld 1997; Charnley 1997) have run which show that even when the oxygen is not initially bound in water, nearly all of the available oxygen is converted into water on a timescale of about one hundred years due to fast neutral-neutral reactions in the warm gas.

The near discontinuity in the water abundance at $T \sim 100 \mathrm{~K}$ is due to the release of water from grain mantles. This discontinuity is consistent with observations of warm $(T \sim 300-500 \mathrm{~K})$ water in absorption toward AFGL 2591 (Helmich et al. 1996; Boonman et al. 2000), with the lack of strong emission by cold water at long wavelengths (Boonman et al. 2000), and by detailed modeling of the line emisson to be discussed in a forthcoming paper (Boonman et al., in preparation).

As noted by Charnley (1997), the ion fraction and electron density grow with time. As seen in Fig. 2 this leads to a destruction of water on timescales of $>10^{5}$ years in the interior, in agreement with the results of Charnley (1997). 
While the cosmic-ray ionization continually creates ions which destroy water, reformation is temperature dependent. A simple extrapolation of the "critical temperature" for water formation from Charnley (1997) for our adopted cosmic-ray ionization rate and density yields $180-200 \mathrm{~K}$. Based upon the temperature structure in Fig. 1, this implies destruction of water for $r \sim 6-8 \times 10^{15} \mathrm{~cm}$, in agreement with the results in Fig. 2. It should be noted that the destruction of water for $t>10^{5}$ yrs is probably unimportant for AFGL 2591 based both upon the water distribution inferred by Boonman et al. (in preparation), and the chemical evolution timescale of $<10^{5}$ yrs discussed in Sect. 5.

Finally, the growth in the water abundance with time in the exterior occurs through slower (due to the lower abundances) ion-molecule reactions. Again, the ionmolecule reactions are driven by cosmic-ray ionization. In the exterior, average abundances of $<3 \times 10^{-7}$ are achieved for $t \sim 3 \times 10^{4}$ years.

The results in Fig. 2 have interesting implications for the interpretation of water abundances. First, a simple estimate of the water abundance inferred from the model radial column densities [assuming $x\left(\mathrm{H}_{2} \mathrm{O}\right)=$ $\left.N\left(\mathrm{H}_{2} \mathrm{O}\right) / N\left(\mathrm{H}_{2}\right)\right]$ would suggest a fractional abundance of water in our model of $x\left(\mathrm{H}_{2} \mathrm{O}\right) \sim 3 \times 10^{-5}$. This is a factor of 5 lower than the actual water abundance adopted in the interior, and would by itself imply a significantly different structure and chemistry involved. This underscores the potential pitfalls in interpreting column densities, as well as the importance of modeling the complete physical, thermal, and chemical structure of the envelope in order to properly compare the relevant regions with observations.

A second implication is that beam dilution can have an important effect on the inferred column densities. A simulated beam-averaged column density commensurate with the beam of the Submillimeter Wave Astronomy Satellite (SWAS) would imply a water abundance of $x\left(\mathrm{H}_{2} \mathrm{O}\right)=$ $N\left(\mathrm{H}_{2} \mathrm{O}\right) / N\left(\mathrm{H}_{2}\right)=10^{-7}-10^{-8}$ depending upon the time considered. This low abundance is due to significant beamdilution from the small region of enhanced $\mathrm{H}_{2} \mathrm{O}$ in the large beam. The range of abundances is similar to that inferred by SWAS (see e.g., Snell et al. 2000; Melnick et al. 2000; Neufeld et al. 2000). Clearly, such an observation alone does not constrain the entire envelope. While it implies that a portion of the envelope (e.g., $T \leq 100 \mathrm{~K}$ ) has a low water abundance, it does not restrict the potential for a compact region of significant water abundance.

As a comparison of the column densities with observations, in Fig. 3 we plot the $\mathrm{H}_{2}, \mathrm{CO}$, and $\mathrm{H}_{2} \mathrm{O}$ column densities as a function of time. We assign errorbars of a factor of two consistent with the intrinsic uncertainties in the $\mathrm{H}_{2} \mathrm{O}$ and $\mathrm{CO}$ results, and with the fact that the $\mathrm{H}_{2}$ results are scaled from the $\mathrm{CO}$ data, as well as various radiative transfer effects. The ranges of the observed column densities are given by the shaded regions. As expected, our data match the observed column densities within the uncertainties.

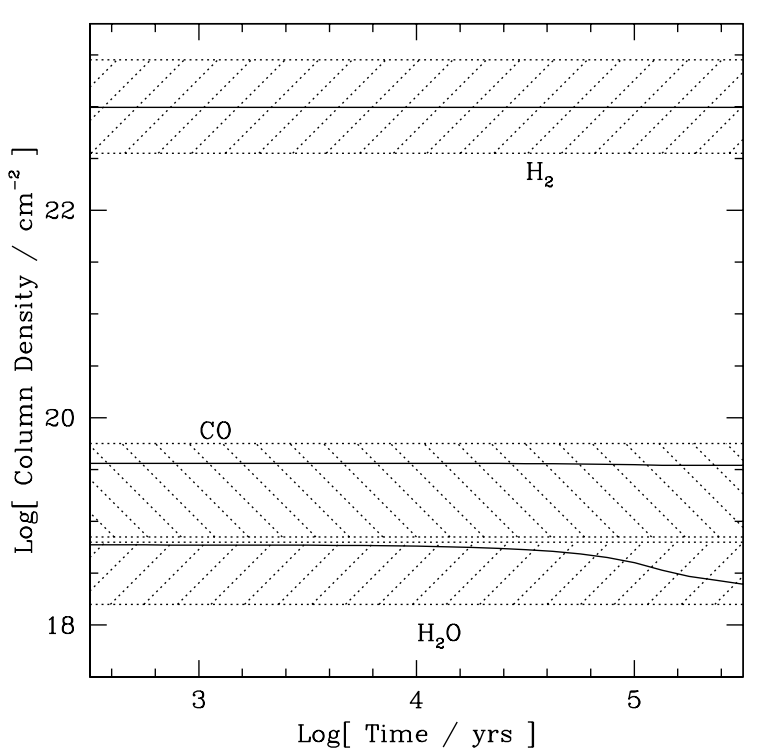

Fig. 3. The radial column densities of $\mathrm{H}_{2}, \mathrm{CO}$, and $\mathrm{H}_{2} \mathrm{O}$ as function of time (solid lines). The shaded regions correspond to the observed abundances (with factor of two errorbars).

\subsection{Hydrocarbon and nitrogen chemistry}

Observations by Lahuis \& van Dishoeck (2000) suggest that the $14 \mu \mathrm{m}$ bands of $\mathrm{C}_{2} \mathrm{H}_{2}$ and $\mathrm{HCN}$ are good tracers of hot gas. Perhaps more importantly, the increase in observed column densities for temperatures above a few hundred $\mathrm{K}$ implies that their chemistry may be altered at high temperatures. Since all of their inferred excitation temperatures are well above the expected desorption temperature of $\sim 100 \mathrm{~K}$, it is expected that these enhanced abundances are due to warm gas-phase chemistry.

In order to test this, we have constructed singleposition models for the chemistry at $n\left(\mathrm{H}_{2}\right)=10^{7} \mathrm{~cm}^{-3}$ and $T \geq 200 \mathrm{~K}$. The results are shown in Fig. 4. Clearly, higher temperatures do increase the abundances of simple hydrocarbons and nitrogen-bearing species, with higher abundances prevalent once $T \sim$ few hundred $\mathrm{K}$.

The enhanced HCN abundance is similar to that found by Rodgers \& Charnley (2001). In parallel with their work, we find that the $756 \mathrm{~K}$ endothermic reaction $\mathrm{CN}+\mathrm{H}_{2} \rightarrow$ HCN proceeds quickly for $T>200 \mathrm{~K}$, producing significant HCN. However, while Rodgers \& Charnley (2001) assume the reaction $\mathrm{C}^{+}+\mathrm{NH}_{3}$ favors $\mathrm{HCNH}^{+}$(following ab initio calculations by Talbi \& Herbst 1998), we assume that $\mathrm{H}_{2} \mathrm{NC}^{+}$is the favored product to account for the observed $\mathrm{HNC} / \mathrm{HCN}$ abundance ratio in many sources. In our case, then, the $\mathrm{CN}$ is formed via the neutral-neutral reaction $\mathrm{CS}+\mathrm{N} \rightarrow \mathrm{CN}+\mathrm{S}$. This reaction has a barrier of $1160 \mathrm{~K}$, leading to significant production for temperatures above $200 \mathrm{~K}$. Overcoming these barriers can increase the abundance from a peak of $10^{-8}$ at $200 \mathrm{~K}$, to $\sim 10^{-7}$ for $t>4 \times 10^{4}$ years, and $\sim 10^{-6}$ for $t>3 \times 10^{5}$ years for $T \geq 400 \mathrm{~K}$.

Methane shows perhaps the most dramatic increase in abundance at very high temperatures. In fact, methane contains more carbon at the latest times than all species 


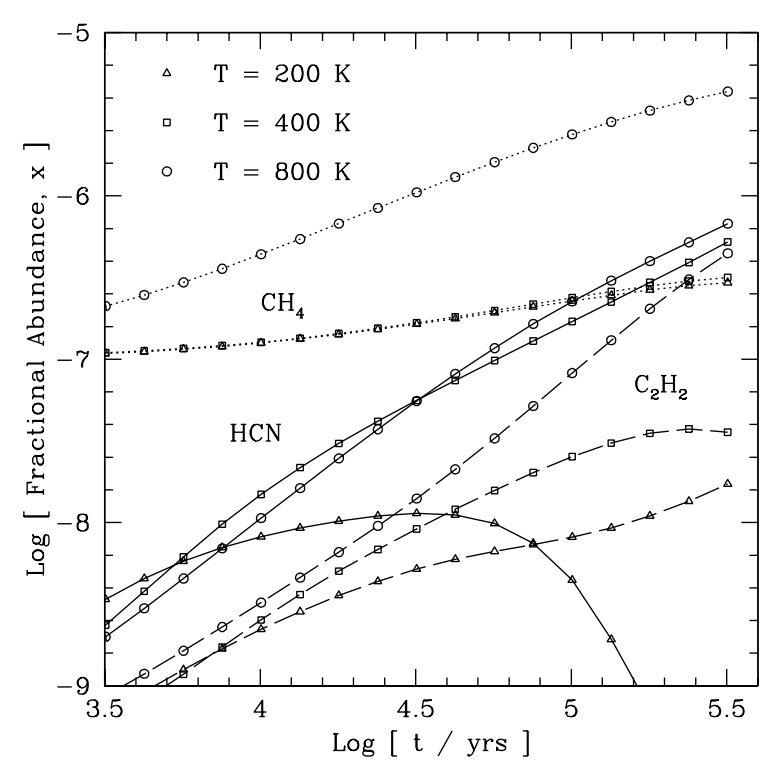

Fig. 4. The fractional abundances of $\mathrm{HCN}, \mathrm{CH}_{4}$, and $\mathrm{C}_{2} \mathrm{H}_{2}$ as functions of time for various temperatures. Here $n\left(\mathrm{H}_{2}\right)=$ $10^{7} \mathrm{~cm}^{-3}$. Notice the general enhancement of the abundances with increasing temperature.

other than $\mathrm{CO}_{2}$ and $\mathrm{CO}$ initially. This is due to the fact that ion-molecule reactions driven by cosmic-ray ionization (e.g., $\mathrm{He}^{+}+\mathrm{CO} \rightarrow \mathrm{C}^{+}+\mathrm{O}$ ) can produce $\mathrm{C}^{+}$. This then reacts via carbon insertion (Herbst 1995) with $\mathrm{H}_{2}$ to form $\mathrm{CH}^{+}$at high temperatures, and then in a chain with $\mathrm{H}_{2}$ up to $\mathrm{CH}_{3}^{+}$, which dissociatively recombines to form CH. While $\mathrm{CH}_{3}^{+}$can also dissociatively recombine to form $\mathrm{CH}_{2}$, the dominant pathway to $\mathrm{CH}_{2}$ at high temperatures is $\mathrm{CH}+\mathrm{H}_{2}+1760 \mathrm{~K} \rightarrow \mathrm{CH}_{2}+\mathrm{H}$. Reactions with $\mathrm{H}_{2}$ then produce $\mathrm{CH}_{4}$ (overcoming barriers of $6400 \mathrm{~K}$ and $4740 \mathrm{~K}$ to form $\mathrm{CH}_{3}$ and $\mathrm{CH}_{4}$ respectively), leading to abundances of $1-3 \times 10^{-7}$ for $T \leq 400 \mathrm{~K}$. However, once the temperature increases to $\sim 600-800 \mathrm{~K}$, abundances can reach $x\left(\mathrm{CH}_{4}\right) \sim 10^{-6}$ at $t \sim 3 \times 10^{4}$ years.

Acetylene is also enhanced at high temperatures. The pathway here is similar to that in diffuse and dark clouds (van Dishoeck \& Hogerheijde 1999). However, in our model, acetylene is formed via reactions of water with $\mathrm{C}_{2} \mathrm{H}_{3}^{+}$instead of dissociative recombination. A second difference is that $\mathrm{C}_{2} \mathrm{H}_{3}^{+}$is produced via $\mathrm{C}_{2} \mathrm{H}_{4}+\mathrm{H}_{3}^{+}$. While the "usual" $\mathrm{CH}_{4}+\mathrm{C}^{+}$production route still occurs, the destruction of $\mathrm{C}_{2} \mathrm{H}_{4}$ by $\mathrm{O}$ is reduced as the temperature increases due to the fact that the oxygen is quickly converted into water by neutral-neutral reactions (see Sect. 4.1). Again, cosmic-ray ionization, carbon insertion, and water play a role, both in the production of $\mathrm{H}_{3}^{+}$, and in the production of $\mathrm{C}_{2} \mathrm{H}_{4}$ via $\mathrm{CO} \rightarrow \mathrm{C}^{+} \rightarrow \ldots \rightarrow \mathrm{CH}_{3}^{+}+\mathrm{CH}_{4} \rightarrow$ $\mathrm{C}_{2} \mathrm{H}_{5}^{+}+\mathrm{H}_{2} \mathrm{O} \rightarrow \mathrm{C}_{2} \mathrm{H}_{4}$. The enhanced $\mathrm{C}_{2} \mathrm{H}_{2}$ abundance is in the range $5 \times 10^{-9} \leq x\left(\mathrm{C}_{2} \mathrm{H}_{2}\right) \leq 2 \times 10^{-8}$ for $200 \mathrm{~K} \leq T \leq 800 \mathrm{~K}$ at $t=3 \times 10^{4}$ years. At late times, it is almost always less than $3 \times 10^{-8}$ at $200 \mathrm{~K}$, less than $5 \times 10^{-8}$ at $400 \mathrm{~K}$, and can reach $5 \times 10^{-7}$ at $800 \mathrm{~K}$.

In order to see how this high-temperature chemistry pertains to our model, in Fig. 5 we plot the fractional

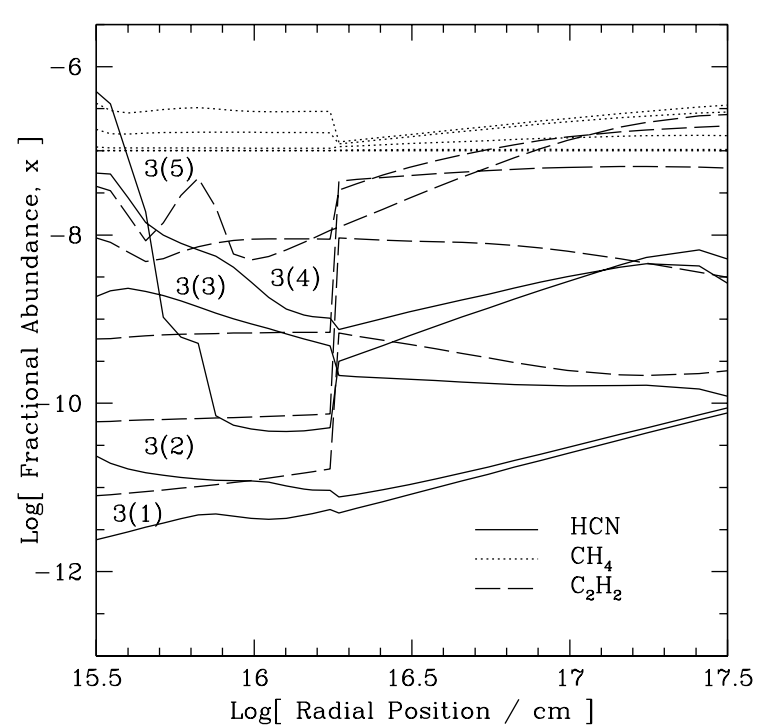

Fig. 5. The time evolution of the fractional abundances of $\mathrm{HCN}, \mathrm{CH}_{4}$, and $\mathrm{C}_{2} \mathrm{H}_{2}$ throughout our model, incorporating the temperature and density distributions desribed in the text. The HCN data are labeled by the time in years, where $a(b)=a \times 10^{b}$. The times for each curve increase upward at the inner radial position. Note the enhancement of the abundances in the warmer interior.

abundances of $\mathrm{HCN}, \mathrm{CH}_{4}$, and $\mathrm{C}_{2} \mathrm{H}_{2}$ throughout the envelope for various times. As expected from the previous discussion, we see enhanced abundances of $\mathrm{HCN}, \mathrm{C}_{2} \mathrm{H}_{2}$, and $\mathrm{CH}_{4}$, especially in the warm interior. The enhancement of $\mathrm{C}_{2} \mathrm{H}_{2}$ in the exterior has two primary causes. First, in this region $\mathrm{C}_{2} \mathrm{H}_{2}$ is primarily formed via dissociative recombination of $\mathrm{C}_{2} \mathrm{H}_{3}^{+}$. The destruction of $\mathrm{C}_{2} \mathrm{H}_{3}^{+}$by $\mathrm{O}$ has a $215 \mathrm{~K}$ barrier that cannot be overcome in the cool exterior, leaving more $\mathrm{C}_{2} \mathrm{H}_{3}^{+}$to produce acetylene. Second, an alternate production pathway via $\mathrm{C}_{3} \mathrm{H}_{3}^{+}+\mathrm{O} \rightarrow \mathrm{C}_{2} \mathrm{H}_{2}$ is enhanced in the exterior due to our increased initial $\mathrm{O}$ abundance in that region (see Table 2).

Cosmic-ray driven ion-molecule chemistry again plays a role for $t>10^{5}$ years. In particular, the destruction of $\mathrm{HCN}$ near $10^{16} \mathrm{~cm}$ is due to reactions with $\mathrm{HCO}^{+}$. For $\mathrm{C}_{2} \mathrm{H}_{2}$ both $\mathrm{HCO}^{+}$and $\mathrm{O}$ are important destruction reactants near $10^{16} \mathrm{~cm}$. The enhancement in $\mathrm{C}_{2} \mathrm{H}_{2}$ near $r \sim 6-8 \times 10^{15} \mathrm{~cm}$ is due to a decrease in atomic oxygen at this position for late times (see also Sect. 4.6).

Observations of high-lying $\mathrm{HCN}$ lines in the submillimeter were undertaken by Boonman et al. (2001). They utilized a sophisticated radiative transfer model of the excitation, line shapes and strengths to analyze their data, and suggested that $\mathrm{HCN}$ follow a "jump" model, with an abundance of $x(\mathrm{HCN}) \sim 1 \times 10^{-6}$ for $T \gtrsim 230 \mathrm{~K}$, and $x(\mathrm{HCN}) \sim 10^{-8}$ for the cool exterior. The results in Figs. 4 and 5 are consistent with this supposition, with abundances of a few $\times 10^{-7}$ at high temperatures, and $\sim 10^{-8}$ at lower temperatures and later times.

As expected, the results in Fig. 5 are not as dramatic as in Fig. 4, as our physical and thermal model only extends into $T \sim 440 \mathrm{~K}$, less than the temperatures at which 


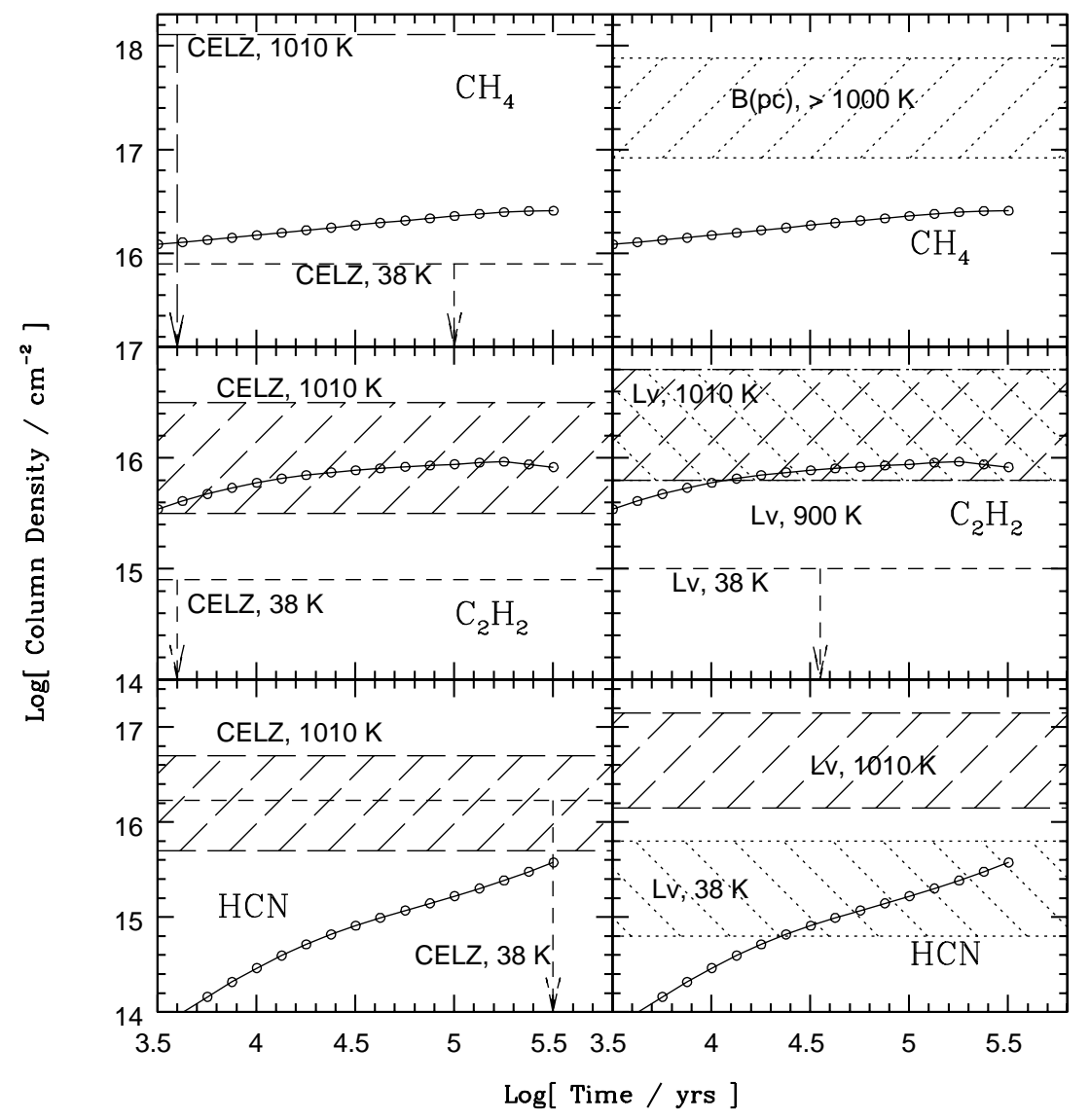

Fig. 6. A comparison of the predicted and observed column densities of $\mathrm{HCN}, \mathrm{CH}_{4}$, and $\mathrm{C}_{2} \mathrm{H}_{2}$ as a function of time. The model predictions are given by the solid lines and accompanied by the filled circles. The observations are divided into two groups. The left-hand panels are for the infrared data of Carr et al. (1995 CELZ), while the right-hand panels are for ISO data from Lahuis \& van Dishoeck (2000 Lv) and Boonman (B(pc), private communication). Data which are upper limits are signified by downward arrows. Other data have been given an arbitrary factor of 3 uncertainty, and are given by the shaded regions.

the greatest enhancements occur. Consequently, care must be used when comparing the results with observations, as the different temperature components may not necessarily probe the portions of the region being modeled.

Such a comparison is given in Fig. 6. Here the model predictions for $\mathrm{CH}_{4}, \mathrm{C}_{2} \mathrm{H}_{2}$, and $\mathrm{HCN}$ are compared with the infrared observational data, which probe column density. In the left-hand panels we compare to the data of Carr et al. (1995), omitting the $200 \mathrm{~K}$ data as these arise in the outflow. In the right-hand panels we compare to the data of Boonman (private communication), and Lahuis \& van Dishoeck (2000).

When we compare with the lower temperature data, the $\mathrm{CH}_{4}$ model results are close to the observed error bounds. On the other hand, they are well above the high temperature component of the observations. This is not a suprise, as the $\mathrm{CH}_{4}$ chemistry is relatively unaffected below about $400 \mathrm{~K}$, with very significant production at higher temperatures.

On the other hand, the $\mathrm{C}_{2} \mathrm{H}_{2}$ data fit the high temperature components of the observations. This seems to imply that while high temperature chemistry can be important, the effects are noticeably smaller than for $\mathrm{CH}_{4}$, consistent with the results of Fig. 5. In particular, the fact that the predicted column density is so much higher than the low-temperature column density suggests that warm chemistry can enhance $\mathrm{C}_{2} \mathrm{H}_{2}$, while the fact that the predicted column densities fall in the lower range of the observed values suggests that there exists room for some enhancement $(\sim 3-5 \times)$ in the $\mathrm{C}_{2} \mathrm{H}_{2}$ abundance at higher temperatures, consistent with Fig. 5 .

Finally, in the lower panels of Fig. 6 we show the comparison for HCN. Here we see the potential for further importance of high-temperature chemistry. In the lower-left panel, the HCN model prediction is consistent with the upper limit derived by Carr et al. (1995) at low temperatures. Similarly, in the lower-right panel, the predicted column densities are consistent with the low-temperature component fit by Lahuis \& van Dishoeck (2000). In both cases, it appears that our model reproduces the production of cool HCN quite well.

On the other hand, the model predictions are well below the observed column densities for the hot components in each of the panels. This is most probably due to the significant production of $\mathrm{HCN}$ at temperatures above $400 \mathrm{~K}$ (see above). This is further supported by the fact that when a single temperature component is determined by Lahuis \& van Dishoeck (2000), they find $T \sim 600 \mathrm{~K}$. Taken 
at face value, their data suggest that our model does not extend inward far enough to include this hot gas.

At this point, one may ask if a simple extension of our power-law model inward would increase the temperature and column density sufficiently to fit the observed HCN data (i.e., at $1010 \mathrm{~K}$ ). We have examined this possibility by extending our model inward, with no success. While a fractional abundance of $x(\mathrm{HCN}) \sim 10^{-7}$ would reproduce the data, the conditions necessary would also produce a water column density $N\left(\mathrm{H}_{2} \mathrm{O}\right) \sim 4 \times 10^{19} \mathrm{~cm}^{-2}$, over an order of magnitude above the observations.

An alternative solution is to adopt a "flattened" (i.e., $\left.n(r) \propto r^{0}\right)$ density profile for $r<r_{\text {in }}$. In this case, the extra column of water would be consistent with the observations, and the column of $\mathrm{HCN}$ would vary as $N(\mathrm{HCN}) \sim$ $2 \times 10^{15}\left(x(\mathrm{HCN}) / 10^{-7}\right)$. While the column could be fit if $x(\mathrm{HCN})=10^{-6}$, this is inconsistent with the results of Fig. 5. First, the chemistry does not show strong variation between 400 and $800 \mathrm{~K}$, suggesting that high temperatures alone will not produce significantly more HCN. Furthermore, to achieve $x(\mathrm{HCN})=10^{-6}$ at these temperatures would require an extended time for chemical evolution in the interior, and would be inconsistent with the abundances of other observed species (see Sect. 5).

There are four possible resolutions to this difficulty. First, and least likely, is the possiblity that the chemical evolution time in the interior is somehow longer than in the exterior. We can think of no way in which this may occur. The second possibility is that the hydrocarbon and nitrogen chemistry is currently incomplete, especially at high temperatures. If another pathway to producing HCN exists above about $600 \mathrm{~K}$, it would be possible to have abundances of $10^{-6}$. Third, there is the possibility that $\mathrm{HCN}$ is present in grain mantles, and is injected into the hot gas. Though this is expected to be unimportant (van der Tak et al. 1999), it may conceivably play a small role.

The fourth, and perhaps most likely, possibility is that there exists some as of yet unidentified destruction mechanism for water at high temperatures. This would remove the problem of the overly-large water column if the envelope were to simply extend further inward. It is possible that evidence exists for this. As discussed by van Dishoeck (1998) observations of water gas and ice toward various sources show significantly less total water in hotter sources than in cooler sources. Given our current understanding of the chemistry of $\mathrm{H}_{2} \mathrm{O}$ production, it would be easiest to explain this effect if there existed a mechanism for $\mathrm{H}_{2} \mathrm{O}$ destruction at high temperatures. Further study into the high temperature chemistry of water, hydrocarbons, and nitrogen-bearing species would be of significant importance in understanding this problem.

\subsection{Sulphur chemistry}

The chemistry of sulphur in hot cores is well-described by Charnley (1997). In our model, we have adopted a chem-

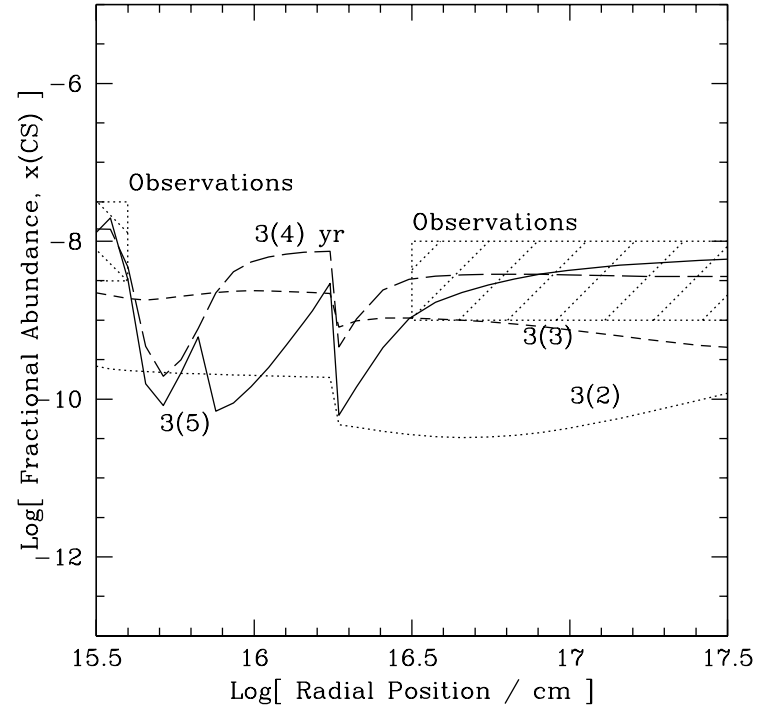

Fig. 7. The fractional abundance of CS throughout the envelope for various times. Nearly half of the sulphur is in CS at late times in the cool exterior, essentially "fixing" the gasphase sulphur abundance. The agreement with observations in the warm interior, however is not fixed. The curves are labeled by the time in years, where $a(b)=a \times 10^{b}$.

istry and set of initial conditions (in the warm region) which is similar. However, given the fact that his model was for a single point in space, while our model extends over a range of physical and thermal parameters, and given recent observations of sulphur-bearing molecules toward AFGL 2591, we present our results here.

In the cool exterior of our model we find that there exist a large number of pathways to shuttle sulphur into CS. The end product is that approximately $50 \%$ of the sulphur is transformed into CS by $t \sim 10^{5}$ years. This is shown in Fig. 7, where we plot the fractional abundance of CS for various times. No single production reaction accounts for more than $25 \%$ of the final CS abundance. This means that, at late times at least, CS is a good measure of the sulphur abundance in the exterior. To accomodate this fact, and in order to match observations of the CS abundance (see Table 1), we adjust the initial sulphur abundance to $x(\mathrm{~S})=6 \times 10^{-9}$ for $T \leq 100 \mathrm{~K}$. This produces a nearly constant abundance in the exterior in good agreement with the observations.

In the interior, the CS abundance increases at intermediate and late times to $x(\mathrm{CS}) \sim 10^{-8}$. This is also in agreement with the observations. However, while the abundance in the exterior is essentially "forced" by our initial sulphur abundance, the fraction in CS in the interior is not.

The variation in the interior CS abundance (as with water and the hydrocarbons) is again related to the oxygen and cosmic-ray driven ion-molecule chemistry. In particular the dip near $r \sim 5 \times 10^{16} \mathrm{~cm}$ is due to the increased atomic oxygen abundance (see Sect. 4.6) in this region. This leads to more conversion of sulphur to $\mathrm{SO}_{2}$, and thus less to CS. The decrease in CS abundance near $10^{16} \mathrm{~cm}$ 
at $t=1-3 \times 10^{5}$ yrs is due to the fact that there is less $\mathrm{OH}$ available for conversion of sulphur out of $\mathrm{H}_{2} \mathrm{~S}$ to $\mathrm{CS}$.

The sulphur abundance in the warm $(T \sim 100-400 \mathrm{~K})$ gas is well-determined by the $\mathrm{SO}_{2}$ abundance. In our model, $\mathrm{SO}_{2}$ is formed by $\mathrm{H}_{2} \mathrm{~S}+(\mathrm{OH}, \mathrm{H}) \rightarrow \mathrm{HS}+\mathrm{O} \rightarrow$ $\mathrm{SO}+\mathrm{OH} \rightarrow \mathrm{SO}_{2}$. The initial reactions of $\mathrm{H}_{2} \mathrm{~S}$ with $\mathrm{H}$ and $\mathrm{OH}$ have barriers of $352 \mathrm{~K}$ and $80 \mathrm{~K}$, respectively. As a result, little $\mathrm{SO}_{2}$ is produced in the cool exterior, while the barriers can be overcome in the interior leading to significant $\mathrm{SO}_{2}$ production. As the temperature further increases, however, the $\mathrm{OH}$ can be more easily forced into water, leaving little for the $\mathrm{SO}+\mathrm{OH} \rightarrow \mathrm{SO}_{2}+\mathrm{H}$ reaction. This can be seen in the very interior of Fig. 8, where the $\mathrm{SO}_{2}$ abundance drops at high temperatures. In our model approximately $90 \%$ of the sulphur returns to atomic form at $\sim 440 \mathrm{~K}$, with approximately $10 \%$ in $\mathrm{H}_{2} \mathrm{CS}$, and a few percent in CS and OCS.

While we are are unable to identify the sulphur reservoir assuming solar abundances roughly hold, it appears that a significant portion would need to exist in or on dust grains. Under this constraint, we can also identify $\mathrm{SO}_{2}$ as the primary sink of molecular sulphur in warm $(100-300 \mathrm{~K})$ gas (assuming no $\mathrm{O}_{2}$ is released during heating of the grain mantles - Charnley 1997). As a result, the sulphur abundance in warm molecular gas at later times can be approximately determined by the $\mathrm{SO}_{2}$ abundance. In our model, this requires the adjustment of the initial $\mathrm{H}_{2} \mathrm{~S}$ abundance from the value of $10^{-7}$ adopted by Charnley (1997) to $1.6 \times 10^{-6}$. This value is, coincidentally, similar to the $\mathrm{H}_{2} \mathrm{~S}$ gas-phase abundance seen by Minh et al. (1990) toward Orion. A comparison of our model predictions with observations by Keane et al. (2001) show similar column densities of $4 \times 10^{16} \mathrm{~cm}^{-2}$ and $6 \times 10^{16} \mathrm{~cm}^{-2}$ respectively. It is also intruiging that the excitation temperature inferred by Keane et al. (2001) for $\mathrm{SO}_{2}$ toward AFGL 2591 is $\sim 750 \mathrm{~K}$, suggesting formation in a warm dense region of a few hundred $K$.

\section{4. $\mathrm{CO}_{2}$ chemistry: Potential heating events?}

An important problem in the chemistry of the envelopes of massive young stars is the low observed gas-phase abundance of $\mathrm{CO}_{2}$ (see e.g., van Dishoeck \& van der Tak 2000). Observations by ISO indicate large solid $\mathrm{CO}_{2}$ abundances (de Graauw et al. 1996; Whittet et al. 1998; Ehrenfreund et al. 1998; Gerakines et al. 1999), with a $\mathrm{CO}_{2} / \mathrm{H}_{2} \mathrm{O}$ abundance in the ice mantles of $10-20 \%$. In the warm regions close to the protostars, these mantles should be evaporated. Assuming water ice abundances of a few $\times 10^{-5}$ (Tielens et al. 1991; Gensheimer et al. 1996) implies a liberated fractional abundance of $x\left(\mathrm{CO}_{2}\right) \sim 10^{-5}-10^{-6}$. On the other hand, ISO observations of gas-phase $\mathrm{CO}_{2}$ (van Dishoeck et al. 1996; Boonman et al. 2000) suggest $x\left(\mathrm{CO}_{2}\right) \sim 10^{-7}$. These results indicate that $\mathrm{CO}_{2}$ is quickly destroyed after evaporation from ice mantles.

To see this discrepancy between the amount of $\mathrm{CO}_{2}$ predicted in our base model and that observed, in Fig. 9

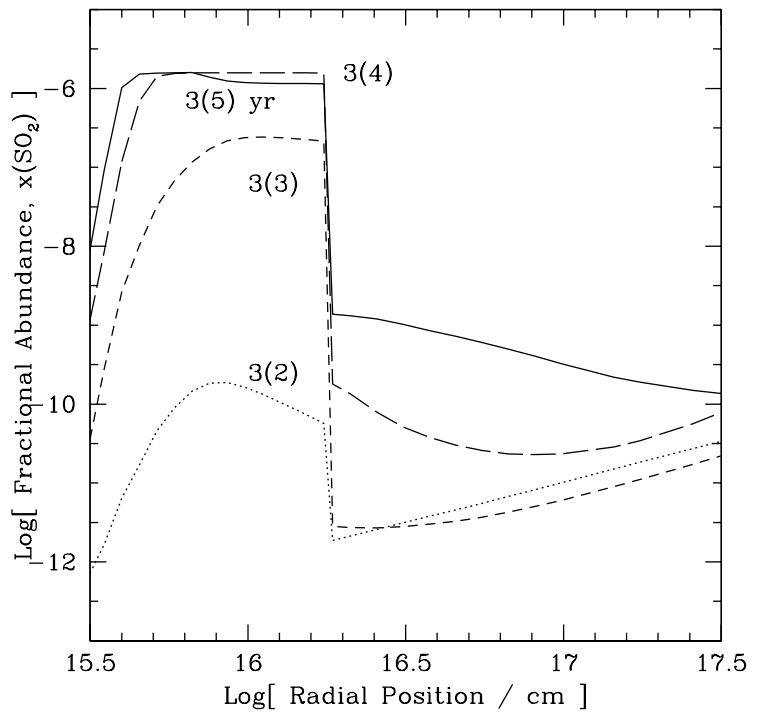

Fig. 8. The fractional abundance of $\mathrm{SO}_{2}$ throughout the envelope at various times. Note the increase near $T=100 \mathrm{~K}$ as the free sulphur is forced into $\mathrm{SO}_{2}$. The decrease at high temperatures is due to the loss of the reactant $\mathrm{OH}$ via its more efficient inclusion into water at those temperatures. The curves are labeled by the time in years, where $a(b)=a \times 10^{b}$.

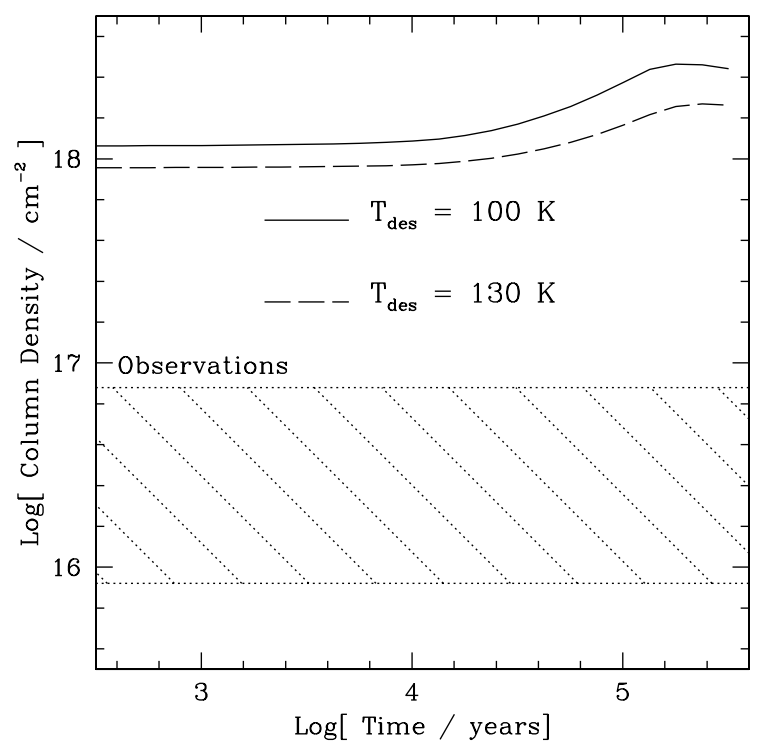

Fig. 9. The column density of $\mathrm{CO}_{2}$ predicted by our base model as a function of time (solid and dashed lines) for two different assumed desorption temperatures. No impulsive heating event is assumed (see text). The observed column is shown by the shaded region. Notice the extent to which the model overpredicts $\mathrm{CO}_{2}$.

we plot the predicted $\mathrm{CO}_{2}$ column density as a function of time. Also plotted are the observations of Boonman et al. (2000). Clearly, the base model significantly overpredicts the $\mathrm{CO}_{2}$ column density in AFGL 2591, confirming the general results above.

Charnley \& Kaufman (2000) studied destruction of $\mathrm{CO}_{2}$ by both $\mathrm{H}$ and $\mathrm{H}_{2}$, suggesting that destruction of $\mathrm{CO}_{2}$ by $\mathrm{H}$ in postshock flows could be important. In order 
to test this, we have constructed models with non-zero atomic hydrogen abundances, as would be expected in partially dissociative shocks. While the $\mathrm{CO}_{2}$ can be effectively destroyed on a shock cooling timescale of $\sim 30 \mathrm{yrs}, \mathrm{CH}_{4}$, $\mathrm{NH}_{3}$, and $\mathrm{H}_{2} \mathrm{O}$ can be destroyed even more efficiently. While this does not pose a significant problem for $\mathrm{CO}_{2}$ or $\mathrm{NH}_{3}$ which have low observed abundances or upper limits, there are effects on other species. In particular, in dissociative shocks the water column density is decreased by a factor of $2-3$. Furthermore, once destroyed, only little water is re-formed in the range $100 \leq T(\mathrm{~K}) \leq 300$, inconsistent with the results of Boonman et al. (in preparation). Similarly, the $\mathrm{O}$ and $\mathrm{O}_{2}$ abundances are significantly increased. On the other hand, the $\mathrm{CH}_{4}$ abundance is decreased by an order of magnitude in the interior. This process only requires a few percent $\mathrm{H}_{2}$ dissociation.

A second potential difficulty is that it is unclear if a large enough fraction of the envelope can be disturbed by a shock to significantly affect the global $\mathrm{CO}_{2}$ abundance, as evidenced by the relatively small line-widths in much of the envelope (van der Tak et al. 1999).

Doty et al. (2002) reconsidered this problem in light of previously unused laboratory measurements of the destruction of $\mathrm{CO}_{2}$ by $\mathrm{H}_{2}$ (Graven \& Long 1954). They found that destruction by $\mathrm{H}_{2}$ may dominate destruction by $\mathrm{H}$ in the very warm gas, near $T \sim 1000-1600 \mathrm{~K}$. While this may occur in a number of ways, Doty et al. (2002) considered two possibilities: a uniform temperature increase (such as from the passage of a $v \sim 20-30 \mathrm{~km} \mathrm{~s}^{-1}$ MHD shock - Draine et al. 1983), and a central luminosity increase caused, for example, by an accretion (FU-Orionistype) event. The possibility of impulsive heating events may be supported by evidence from continuum emission by crystalline silictes (Smith et al. 2000; Aitken et al. 1988) which suggests that an annealing event may have occurred in AFGL 2591. If such a heating event occurred, Doty et al. (2002) find that it is possible for the $\mathrm{CO}_{2}$ to be removed on a timescale of $10^{0}-10^{4}$ years by $\mathrm{H}_{2}$. Recent calculations of the potential surface for the $\mathrm{CO}_{2}+\mathrm{H}_{2}$ reaction suggest, however, that the barrier for the reaction may be higher than indicated by the old laboratory experiments, so that this issue remains unsettled (Talbi \& Herbst 2002). Clearly, further laboratory studies of this reaction at high temperatures are urgently needed.

While speculative, destruction of $\mathrm{CO}_{2}$ by $\mathrm{H}_{2}$ in this fashion has some advantages. First, there is very little atomic hydrogen available to affect the chemistry, and in particular to influence $\mathrm{CH}_{4}, \mathrm{O}, \mathrm{O}_{2}$, and $\mathrm{H}_{2} \mathrm{O}$. Second, and perhaps more importantly, variations in the observed column density of $\mathrm{CO}_{2}$ may potentially be explained by variations in the size and/or duration of the proposed heating event - depending upon its origin, or the time since the heating event and the local cosmic-ray ionization rate.

As a final note, it is interesting to also consider the possibility that the $\mathrm{CO}_{2}$ desorption temperature may be greater than 100 K. Recent work by Fraser et al. (2001), suggests that the desorption temperature of water may be as high as $120-130 \mathrm{~K}$. If the solid $\mathrm{CO}_{2}$ is contained in a water-ice matrix as suggested by observations (Gerakines et al. 1999), then it may be interesting to consider the effect of this higher desorption temperature on $N\left(\mathrm{CO}_{2}\right)$. In Fig. 9, we present predicted column densities for the re-formation of $\mathrm{CO}_{2}$, assuming desorption temperatures of both $100 \mathrm{~K}$ and $130 \mathrm{~K}$. The effect is a decrease in the $\mathrm{CO}_{2}$ column densities by a factor of two, insufficient to explain the discrepancies.

\subsection{Cosmic-ray ionization rate}

As discussed earlier, cosmic-ray ionization can play an important role in driving ion-molecule chemistry at later times. In our model, we adopt the cosmic-ray ionization rate for AFGL 2591 of $\zeta=5.6 \times 10^{-17} \mathrm{~s}^{-1}$ as determined by van der Tak \& van Dishoeck (2000). While the cosmic ray flux is unique, the ionization rate will vary with position if the particles are absorbed. As evidence for cosmic ray absorption is inconclusive (see, e.g., van der Tak 2002), we adopt a single cosmic ray ionization rate for AFGL 2591.

In Fig. 10 we plot the predicted fractional abundance of $\mathrm{HCO}^{+}$and $\mathrm{N}_{2} \mathrm{H}^{+}$. There are two important features. First, there is significant destruction of $\mathrm{HCO}^{+}$at the water desorption position, due to the reaction $\mathrm{HCO}^{+}+\mathrm{H}_{2} \mathrm{O} \rightarrow$ $\mathrm{H}_{3} \mathrm{O}^{+}+\mathrm{CO}$. This is in agreement with the model of van der Tak \& van Dishoeck (2000). While they argue that this jump in abundances is not important in constraining the cosmic ray ionization rate, our overall $\mathrm{HCO}^{+}$abundance is consistent with their observations, and thus lends support to their somewhat high value for $\zeta$ in AFGL 2591. The situation is similar for $\mathrm{N}_{2} \mathrm{H}^{+}$. Second, at $t=3 \times 10^{5}$ yrs, the ion abundances increase in the interior. This is consistent with Charnley (1997), and is due to the fact that the cosmic-ray ionization continues to produce more ions, which eventually destroy a significant fraction of the complex molecules up to the position where the temperature is high enough to re-form them.

The cosmic-ray ionization rate also affects the abundance of $\mathrm{H}_{3}^{+}$. In our models, reasonable time $(t \geq$ $10^{3}$ years) column densities are $\sim 5 \times 10^{14} \mathrm{~cm}^{-2}$, almost a factor of 5 below those observed by McCall et al. (1999). If a comparison of these results were used to infer a cosmicray ionization rate, one would obtain a much larger value. While large $\mathrm{H}_{3}^{+}$abundances in the diffuse ISM have been reported by McCall et al. (2002) - which they suggest may be due to uncertainties in dissociative recombination rate - van der Tak \& van Dishoeck (2000) have also noted that there exists a variation in $\mathrm{H}_{3}^{+}$column density with distance which suggests that intervening clouds may be important.

The cosmic-ray ionization rate also affects the HCN abundance. Decreasing $\zeta$ makes it harder to form HCN. For example lowering $\zeta$ by a factor of three decreases the enhancement of $\mathrm{HCN}$ by a factor of three even at $800 \mathrm{~K}$, placing the warm HCN abundance at $\leq 3 \times 10^{-7}-$ well below the observations. Furthermore, 


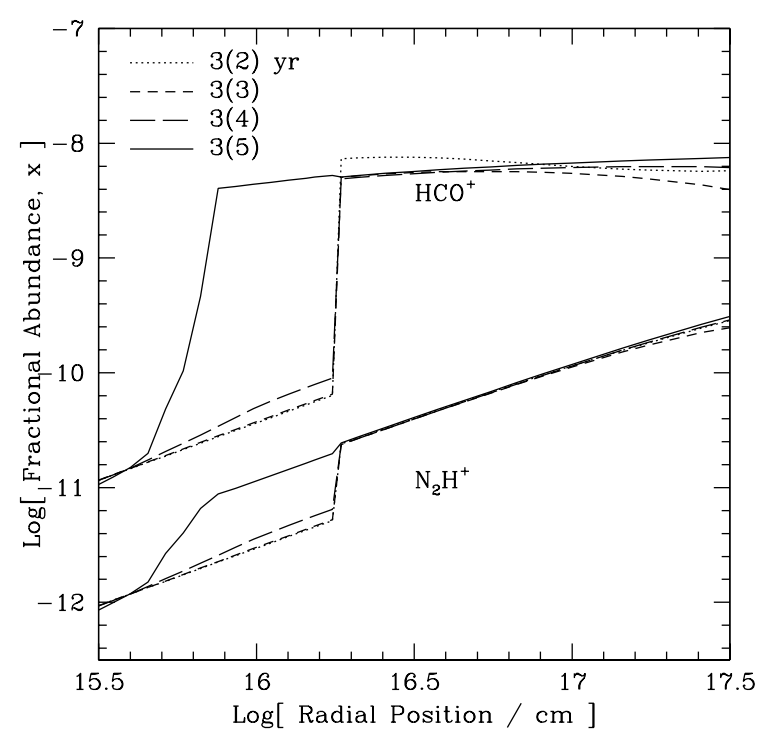

Fig. 10. The fractional abundance of $\mathrm{HCO}^{+}$and $\mathrm{N}_{2} \mathrm{H}^{+}$ througout the envelope for various times. Note the marked decrease about $100 \mathrm{~K}$, where reactions with $\mathrm{H}_{2} \mathrm{O}$ become important. The curves are labeled by the time in years, where $a(b)=a \times 10^{b}$.

the same change also increases the time for the cold column of $\mathrm{HCN}$ to reach the observed range to $t \sim 10^{5} \mathrm{yrs}$, in disagreement with the age constraints discussed below in Sect. 5.

Based upon these results, it appears that the value of $\zeta=5.6 \times 10^{-17} \mathrm{~s}^{-1}$ inferred by van der Tak \& van Dishoeck (2000) is correct to within a factor of three. Any value much lower would significantly hamper the production of HCN, making for disagreement with the observations. Any value much higher would be in conflict with the observed ion abundances.

\subsection{Other species}

As oxygen and oxygen-bearing species can have a signifcant effect on the chemistry, in Fig. 11 we plot the fractional abundances of $\mathrm{O}, \mathrm{OH}$, and $\mathrm{O}_{2}$ as functions of position for various times.

The increase in the atomic oxygen abundance near $10^{16} \mathrm{~cm}$ is due to the fact that $\mathrm{O}$ is freed from water at late times via ion-molecule reactions with $\mathrm{H}_{2} \mathrm{O}$ and $\mathrm{CO}$ as discussed in Sect. 4.1. As the water is destroyed, the main production mechanism for $\mathrm{OH}\left[\left(\mathrm{HCO}^{+}, \mathrm{H}_{3}^{+}\right)+\mathrm{H}_{2} \mathrm{O} \rightarrow\right.$ $\left.\mathrm{H}_{3} \mathrm{O}^{+}+\mathrm{e}^{-} \rightarrow \mathrm{OH}\right]$ is removed. This leads to a decreased $\mathrm{OH}$ abundance at this position at late times.

The peak in the atomic oxygen abundance near $r \sim$ $5-8 \times 10^{15} \mathrm{~cm}$ is due to the competition between production of $\mathrm{O}$ by ion-molecule reactions with $\mathrm{CO}$, and the destruction of $\mathrm{O}$ at high temperatures by reactions with $\mathrm{OH}$ and $\mathrm{H}_{2}$. Once the temperature reaches $\sim 180-200 \mathrm{~K}$, neutral-neutral re-formation of water can balance the destruction by ion-molecule reactions on these timescales, as discussed in Sect. 4.1, and in Fig. 2. This leads to a greater $\mathrm{OH}$ abundance at these positions, and thus a decreased $\mathrm{O}$ abundance.

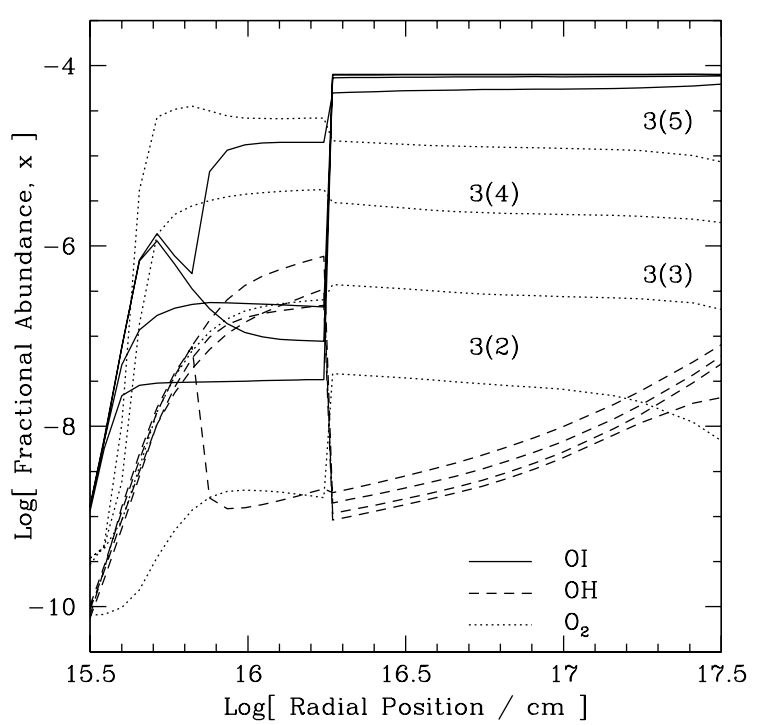

Fig. 11. The fractional abundance of $\mathrm{O}, \mathrm{OH}$, and $\mathrm{O}_{2}$ throughout the envelope for various times. The curves are labeled by the time in years, where $a(b)=a \times 10^{b}$. The times increase upward. The only exception is the dip for $\mathrm{OH}$ near $10^{16} \mathrm{~cm}$, which corresponds to $t=3 \times 10^{5}$ yrs.

In any case, the excess atomic oxygen is easily converted to molecular oxygen over time at temperatures less than $300 \mathrm{~K}$. This places an important constraint on the temporal evolution of the source as discussed in Sect. 5 below.

It is also interesting to note that the dominant nitrogen resevoir is molecular nitrogen. While atomic nitrogen is somewhat abundant (see Table 3 ), only about $1 \%$ or less of the nitrogen is in atomic form - and that preferentially at later times.

Although we have endeavored to consider detailed comparisons between our model predictions and observations, a worthwhile test of any model is the predictions it makes for future observations. Consequently, in Table 3, we give predicted radial and beam-averaged column densities at $t=3 \times 10^{4}$ yrs for various species with $N>10^{13} \mathrm{~cm}^{-2}$. The beam-averaged column densities assume a Gaussian beam of full-width at half-max of 15 arcsec, though the results are insensitive to this assumption.

\subsection{Implications for mantles and mantle destruction}

Finally, although we do not explicitly consider grainsurface chemistry, it is worthwhile to discuss the implications our results have on the grain mantles, and grainsurface chemistry. Two observed species that may form on grain mantles are $\mathrm{H}_{2} \mathrm{CO}$ and $\mathrm{CH}_{3} \mathrm{OH}$. In Fig. 12 we plot the fractional abundance of $\mathrm{H}_{2} \mathrm{CO}$ and $\mathrm{CH}_{3} \mathrm{OH}$ throughout the envelope at various times.

When low-temperature depletion is assumed, the column densities for $\mathrm{H}_{2} \mathrm{CO}$ and $\mathrm{CH}_{3} \mathrm{OH}$ are generally close to the observations. In particular, while van der Tak et al. (2000) report column densities of $N\left(\mathrm{H}_{2} \mathrm{CO}\right)=$ $8 \times 10^{13} \mathrm{~cm}^{-2}$ and $N\left(\mathrm{CH}_{3} \mathrm{OH}\right)=1.2 \times 10^{15} \mathrm{~cm}^{-2}$ 
Table 3. Predicted column densities at $t=3 \times 10^{4} \mathrm{yrs}$.

\begin{tabular}{|c|c|c|}
\hline Species & $N_{\text {radial }}(\mathrm{X})$ & $N_{\text {beam }}(\mathrm{X})-15^{\prime \prime}$ \\
\hline OI & $4(18)$ & $3(18)$ \\
\hline $\mathrm{N}$ & $3(16)$ & $2(16)$ \\
\hline S & $1(16)$ & $7(13)$ \\
\hline $\mathrm{NO}$ & $1(16)$ & $5(15)$ \\
\hline $\mathrm{OH}$ & $9(15)$ & $1(15)$ \\
\hline $\mathrm{SO}$ & $4(15)$ & $2(13)$ \\
\hline $\mathrm{H}_{2} \mathrm{CS}$ & $2(15)$ & $1(13)$ \\
\hline $\mathrm{C}_{3} \mathrm{H}$ & $2(15)$ & $2(15)$ \\
\hline $\mathrm{C}_{4} \mathrm{H}$ & $1(15)$ & $1(15)$ \\
\hline $\mathrm{C}_{3} \mathrm{H}_{2}$ & $7(14)$ & $7(14)$ \\
\hline $\mathrm{CH}_{3} \mathrm{OCH}_{3}$ & $6(14)$ & $7(12)$ \\
\hline $\mathrm{CHOOH}$ & $4(14)$ & $1(14)$ \\
\hline $\mathrm{NH}_{2}$ & $3(14)$ & $2(13)$ \\
\hline $\mathrm{CH}_{2} \mathrm{CO}$ & $2(14)$ & $2(14)$ \\
\hline $\mathrm{CH}_{3}$ & $2(14)$ & $6(12)$ \\
\hline $\mathrm{NH}$ & $1(14)$ & $3(12)$ \\
\hline $\mathrm{H}_{3} \mathrm{O}^{+}$ & $8(13)$ & $2(13)$ \\
\hline $\mathrm{CN}$ & $5(13)$ & $5(13)$ \\
\hline $\mathrm{OCN}$ & $5(13)$ & $1(13)$ \\
\hline NS & $5(13)$ & $9(10)$ \\
\hline $\mathrm{C}_{2} \mathrm{~S}$ & $4(13)$ & $3(13)$ \\
\hline $\mathrm{HS}_{2}$ & $4(13)$ & $1(12)$ \\
\hline $\mathrm{C}_{6} \mathrm{H}$ & $4(13)$ & $4(13)$ \\
\hline $\mathrm{C}_{3} \mathrm{H}_{3}$ & $3(13)$ & $2(13)$ \\
\hline $\mathrm{CCN}$ & $3(13)$ & $3(13)$ \\
\hline $\mathrm{H}_{2} \mathrm{C}_{3}$ & $3(13)$ & $2(13)$ \\
\hline $\mathrm{CH}_{3} \mathrm{OH}_{2}^{+}$ & $2(13)$ & $2(11)$ \\
\hline $\mathrm{HNO}$ & $2(13)$ & $1(13)$ \\
\hline $\mathrm{CH}_{3} \mathrm{CHO}$ & $2(13)$ & $7(12)$ \\
\hline
\end{tabular}

$a(b)$ means $a \times 10^{b}$.

All column densities given in $\mathrm{cm}^{-2}$.

respectively, the model predicts a $\mathrm{CH}_{3} \mathrm{OH}$ column density about 6 times lower, and an $\mathrm{H}_{2} \mathrm{CO}$ column density about 5 times higher.

On the other hand, detailed radiative transfer modeling by van der Tak et al. (2000) suggests that the observed lines are consistent with a uniform $\mathrm{H}_{2} \mathrm{CO}$ abundance of $4 \times 10^{-9}$, and a $\mathrm{CH}_{3} \mathrm{OH}$ abundance of $2.6 \times 10^{-9}$ for $T \leq 100 \mathrm{~K}$, and $8 \times 10^{-8}$ for $T \geq 100 \mathrm{~K}$. For comparison the predicted abundances for these species are shown in Fig. 12. The $\mathrm{H}_{2} \mathrm{CO}$ abundance in the cool exterior is consistent with the inferred abundance, while the abundance in the warm interior is predicted to be significantly higher and decreases only slowly with time. The $\mathrm{CH}_{3} \mathrm{OH}$, on the other hand, does not fit the inferred abundances very well. While there does exist a "jump" as suggested by van der Tak et al. (2000), the abundances predicted by the model are significantly too low in the exterior and too high in the interior.

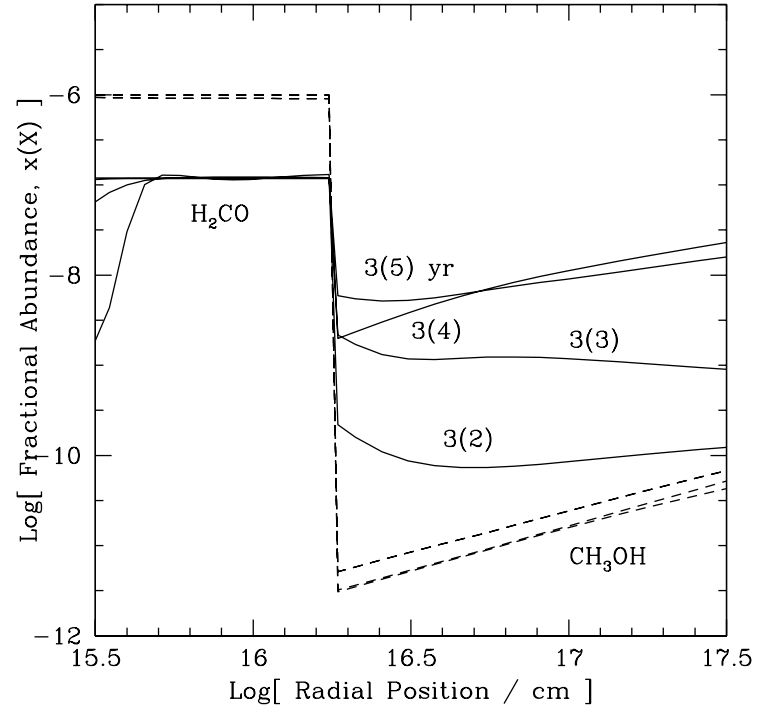

Fig. 12. The fractional abundance of $\mathrm{H}_{2} \mathrm{CO}$ and $\mathrm{CH}_{3} \mathrm{OH}$ throughout the envelope for various times. The curves are labeled by the time in years, where $a(b)=a \times 10^{b}$. Note that both species are initially depleted from the gas phase for $T \leq 100 \mathrm{~K}$.

For comparison, we have also run models where the abundances of $\mathrm{H}_{2} \mathrm{CO}$ and $\mathrm{CH}_{3} \mathrm{OH}$ are initially undepleted for $T \leq 100 \mathrm{~K}$. In models where the cold initial abundances of $\mathrm{CH}_{3} \mathrm{OH}$ are set equal to the hot initial abundances only $\mathrm{CH}_{3} \mathrm{OH}, \mathrm{C}_{3} \mathrm{H}_{3}$, and $\mathrm{CH}_{3} \mathrm{OCH}_{3}$ show column density differences of a factor of three or more. When we adopt this initial abundance, the column density of $\mathrm{CH}_{3} \mathrm{OH}$ increases to $4.4 \times 10^{15} \mathrm{~cm}^{-2}$, a value approximately 4 times larger than the observations. These results suggest that while we can reproduce the $\mathrm{CH}_{3} \mathrm{OH}$ column density with some accuracy, simple gas-phase chemistry alone cannot reproduce the apparent details of the $\mathrm{CH}_{3} \mathrm{OH}$ abundance distribution. As a result, it appears that $\mathrm{CH}_{3} \mathrm{OH}$ can be strongly affected by grain surface chemistry, also in the cooler regions.

Allowing a cold $\mathrm{H}_{2} \mathrm{CO}$ abundance equal to the warm abundance changes the predicted column density by only a factor of two, with only minor differences for all other species. This implies that only observations of high-enough spatial resolution to differentiate between the warm and cold phases, or use of high-excitation lines will be able to best determine the nature of $\mathrm{H}_{2} \mathrm{CO}$ formation.

It is also interesting to consider the fact that submillimeter observations suggest that the abundances of warm $\mathrm{H}_{2} \mathrm{CO}$ and $\mathrm{CH}_{3} \mathrm{OH}$ are factors of 100-1000 below the solid state abundances. In our models, we find that the ratio of warm to cold $\mathrm{H}_{2} \mathrm{CO}$ for a 15 arcsec beam is $60 \leq\left[N\left(\mathrm{H}_{2} \mathrm{CO}\right)\right]_{T \geq 100} /\left[N\left(\mathrm{H}_{2} \mathrm{CO}\right)\right]_{T \leq 100} \leq 500$ for $3 \times 10^{4} \leq t(\mathrm{yrs}) \leq 10^{5}$. On the other hand, for $\mathrm{CH}_{3} \mathrm{OH}$ we find that $1 \leq\left[N\left(\mathrm{CH}_{3} \mathrm{OH}\right)\right]_{T \geq 100} /\left[N\left(\mathrm{CH}_{3} \mathrm{OH}\right)\right]_{T \leq 100} \leq 2$ for $3 \times 10^{4} \leq t(\mathrm{yrs}) \leq 10^{5}$. This confirms the previous suggestions that that gas-phase chemistry may perhaps dominate grain-surface chemistry in the production of $\mathrm{H}_{2} \mathrm{CO}$, while there must be some other (presumeably grain-surface) pathway to the production of $\mathrm{CH}_{3} \mathrm{OH}$. 


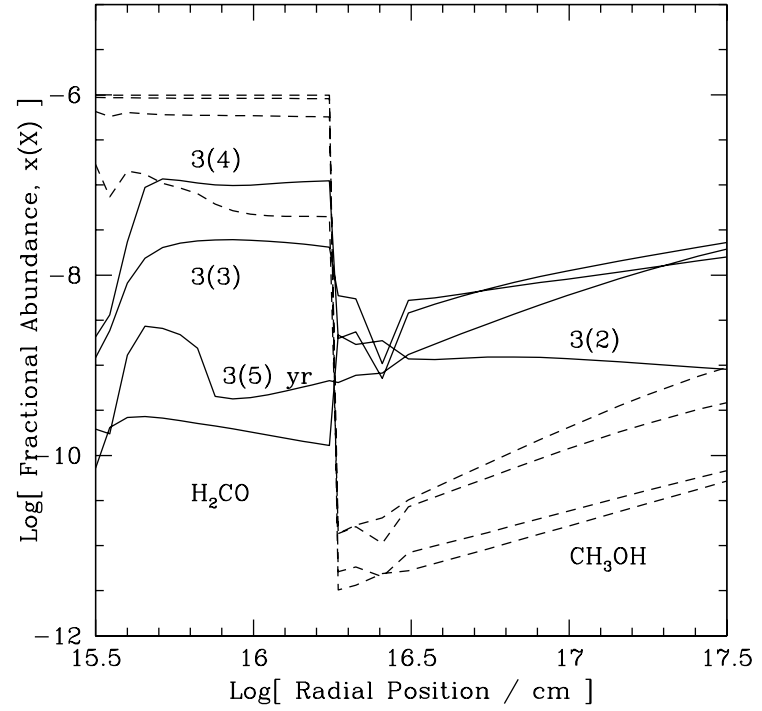

Fig. 13. The fractional abundance of $\mathrm{H}_{2} \mathrm{CO}$ and $\mathrm{CH}_{3} \mathrm{OH}$ throughout the envelope for various times, after a hypothetical non $\mathrm{H}_{2}$-dissociative heating event. The curves are labeled by the time in years, where $a(b)=a \times 10^{b}$. Note that both species are initially depleted from the gas phase for $T \leq 100 \mathrm{~K}$.

As a final note, we have considered the effect of a heating event (as proposed in Sect. 4.4) in which $\mathrm{H}_{2}$ is not dissociated on the $\mathrm{H}_{2} \mathrm{CO}$ and $\mathrm{CH}_{3} \mathrm{OH}$ chemistry. The results are shown in Fig. 13. As can be seen, the later-time abundances are more consisent with the results inferred by van der Tak et al. (2000). In particular, the $\mathrm{CH}_{3} \mathrm{OH}$ abundance in the interior is in the range of $5 \times 10^{-8} \leq x\left(\mathrm{CH}_{3} \mathrm{OH}\right) \leq 6 \times 10^{-7}$, while in the exterior the abundances can reach as high as $0.3-1 \times 10^{-9}$. Likewise, the $\mathrm{H}_{2} \mathrm{CO}$ abundance for $3 \times 10^{4} \leq t(\mathrm{yrs}) \leq 3 \times 10^{5}$ is in the range $10^{-7} \geq x\left(\mathrm{H}_{2} \mathrm{CO}\right) \geq \overline{3} \times 10^{-10}$. While not conclusive, this brackets the inferred $\mathrm{H}_{2} \mathrm{CO}$ abundance of $4 \times 10^{-9}$ nicely. If further suggestions of a heating event are found, it may be useful to re-visit these data for comparison with observations as they may provide a gas-phase mechanism for the production of $\mathrm{H}_{2} \mathrm{CO}$ and $\mathrm{CH}_{3} \mathrm{OH}$.

\section{Time constraints}

Based upon the large amount of observational data for AFGL 2591 (see Table 1), and given the time-dependent nature of the reaction network, one important test of the physical and chemical model would be a determination of the chemical evolution time of the envelope, consistent with all or most of the observed species. This has been proposed and carried out previously (e.g., Stahler 1984; Millar 1990; Helmich et al. 1994; Hatchell et al. 1998) in singlepoint models of dense cloud cores, with some success.

In our case, we determine the time-dependent fractional abundances and column densities for each of the species observed in Table 1. As discussed in Sect. 2.3, we divide the data into two sets: those for which infrared absorption measurements have been made, and those for

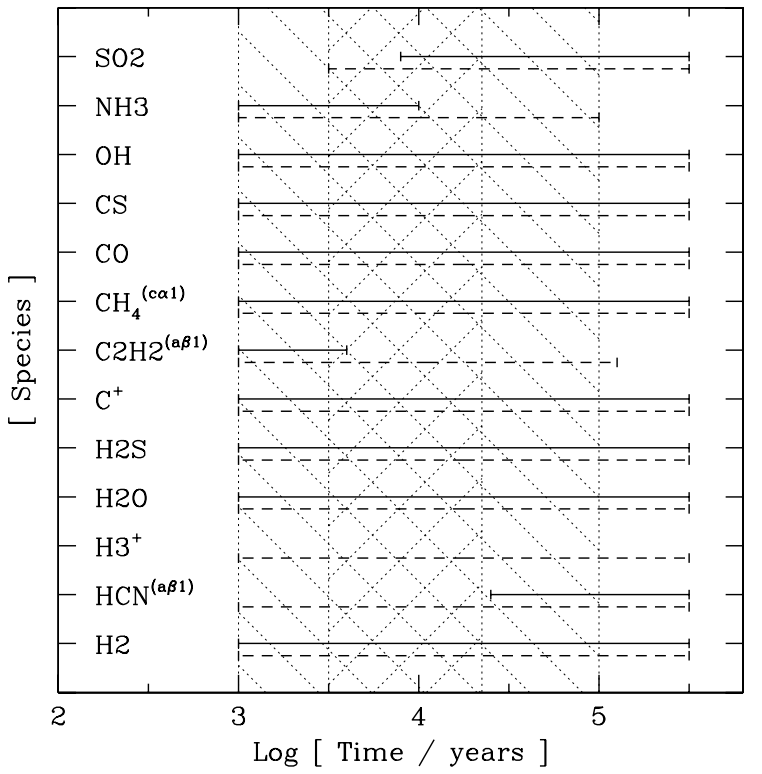

Fig. 14. A comparison of the predicted and observed abundances and column densities for the species listed in Table 1, and observed in the infrared (see text). The solid lines correspond to agreement between the models and observations within a factor of 3 , and the dashed-lines to within a factor of 10 . The species are listed, with notes on the observational fits given as parentheses as in Table 1. The two shaded regions denote the regions of potential and preferred fit between the model and the observations (see text). Notice the agreement with Fig. 15.

which submillimeter emission measurements have been made.

In Figs. 14 and 15 we plot the approximate time ranges over which the listed species match the observed data. In both figures, the solid lines represent agreement to within a factor of 3, while the dashed lines represent agreement to within a factor of 10 . These limits can be considered good and acceptable levels of agreement respectively (see e.g., Millar \& Freeman 1984, and Brown \& Charnley 1990). In both figures, the lower limits to the chemical evolution time are capped at $10^{3}$ years. As in Table 1 , observational data are appended to each species name. We include all species from Table 1 , except for $\mathrm{CO}_{2}$. As discussed in Sect. 4.4, there are significant discrepancies and questions about the gas-phase chemistry of $\mathrm{CO}_{2}$, and as such we have treated it separately in that section. For species other than $\mathrm{CO}_{2}$, we include the data that is relevant to comparison with our model (i.e., the most reliable components).

In Fig. 14, we can see a wide variation of possible times when constraining the models by the infrared data. We place two limits on the evolution: a wide range of $10^{3} \leq$ $t(\mathrm{yrs}) \leq 1 \times 10^{5}$, and a preferred limit of $3 \times 10^{3} \leq t(\mathrm{yrs}) \leq$ $2.5 \times 10^{4}$. These regions are identified by the shading in Fig. 14.

For comparison, in Fig. 15 we plot the constraints on the time for the submillimeter data. As discussed in Sect. 2.3, results based upon sophisticated, self-consistent, radiative transfer modeling are given slightly more weight, 


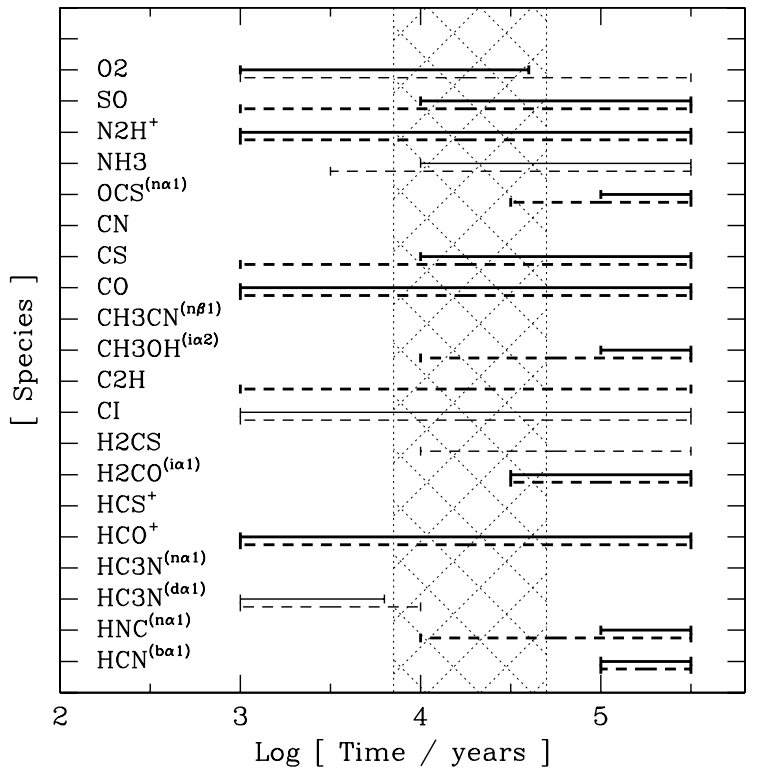

Fig. 15. A comparison of the predicted and observed abundances and column densities for the species listed in Table 1, and observed in the submillimeter (see text). The solid lines correspond to agreement between the models and observations within a factor of 3, and the dashed-lines to within a factor of 10 (see text for details). The species are listed, with notes on the observational fits given as parentheses as in Table 1. The shaded region denote the regions of preferred fit between the model and the observations. Notice the agreement with Fig. 14.

and identified by the bold lines in Fig. 15. Also, the $\mathrm{O}_{2}$ upper limit by SWAS is given more weight. This is done because in the absence of upper limits for the $\mathrm{O}_{2}$ abundance toward AFGL 2591 in particular, we have used the largest quoted upper limit of $N\left(\mathrm{O}_{2}\right) / N\left(\mathrm{H}_{2}\right) \leq 9 \times 10^{-7}$ (Goldsmith et al. 2000). Where radiative-transfer modeling derived abundances are not available, we have calculated the appropriate beam-averaged column densities for comparison with the observations.

Most species in Fig. 15 fit the models to within an order of magnitude of the observational data. Those that do not fit are not significant defects, for a number of reasons. First, not all data are in disagreement with the models both OCS and $\mathrm{HC}_{3} \mathrm{~N}$ have other observations / reductions which do agree with the models. Second, the discrepancies can be understood on a case-by-case basis. For instance, the chemistry and reaction rates of OCS are only poorly understood at best (Millar, private communication). It is interesting to note, however, that the radial OCS column density matches the observed column density. Also, we expect the potential difficulties with species related to $\mathrm{HCN}$ (such as $\mathrm{HC}_{3} \mathrm{~N}$ and $\mathrm{CH}_{3} \mathrm{CN}$ ) as our model does not probe the complete region over which significant $\mathrm{HCN}$ production may be important (see Sect. 4.2 above). In the case of $\mathrm{HCS}^{+}$, the abundance is strongly affected by enhancements of CS abundance at temperatures of 10-20 K (Helmich 1996), lower than all but our outermost temperature, signifying that the AFGL 2591 envelope may be more extended than we have assumed. In a similar

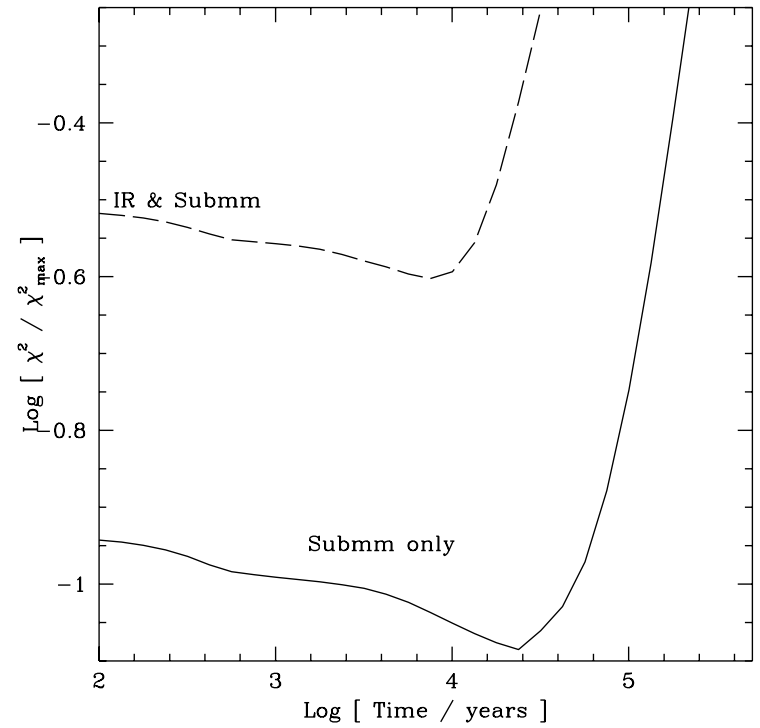

Fig. 16. The quality of the model fit to the observations as a function of time. Here we plot the $\chi^{2}$ as defined in the text, normalized to the maximum value for the times considered. The solid line shows the chi-squared for the submillimeter data only, while the dashed-line shows the results when both the submillimeter and infrared data are included.

fashion, CN is strongly influenced by UV radiation from a PDR (Helmich 1996), a radiation source not considered in our model.

The age-constraints implied by the results in Fig. 15 suggest chemical evolution times in the range $7 \times 10^{3} \leq$ $t(\mathrm{yrs}) \leq 5 \times 10^{4}$, with a strong preference for $t \sim 3 \times$ $10^{4}$ years. These constraints are shown by the shaded region in the figure.

In order to attempt to quantify this result, in Fig. 16 we plot the chi-squared value between the models and the observations, normalized to the maximum chi-squared in the entire time evolution. The $\chi^{2}$ is defined by $\chi^{2} \equiv$ $\Sigma_{i} w_{i}^{2} \times\left[y_{\text {model }, i}-y_{\mathrm{obs}, i}\right]^{2} / \sigma_{i}^{2}$. Here we include the weight from Table 1 as $w$, and assume uncertainties of a factor of 5 in the observations for all species except $\mathrm{O}_{2}$, for which we assume a factor of 2 uncertainty as we already have adopted the highest observed upper limit from Goldsmith et al. (2000). These results are generally consistent with those of Figs. 14 and 15, both in terms of the preferred times as well as the relatively lower level of constraint provided by the IR data. In particular, while times of up to $\sim 10^{5}$ yrs may be possible, there appears to be a preference for somewhat lower times near $\sim 3 \times 10^{4}$ yrs.

It is interesting and reassuring that the chemical evolution times from both the infrared and submillimeter data provide similar results. This is especially true as they probe such different regions of the envelope. Also, the nearly simultaneous agreement in time between such different species, with transitions arising throughout the envelope, and observed with a range of ground- and space-based instruments provides significant support to the proposed chemical, physical, and thermal structure of the envelope. 


\section{Conclusions}

We have constructed detailed thermal and gas-phase chemical models for AFGL 2591 based upon the physical model of van der Tak et al. (1999, 2000). These models were used to probe the validity of the proposed physical structure, as well as study the chemical evolution of the source.

In particular, we find that:

1. Pseudo-time dependent modeling of the chemistry as a function of depth is a good probe of the physical and thermal structure of the source, due to the density and temperature dependence of the chemistry.

2. Care must be exercised when drawing conclusions from observations, as infrared absorption measurements usually better probe the warm interior of the envelope while submillimeter emission data often better probe the cool exterior. While "average abundances" $\left[x_{\text {avg }} \equiv N(\mathrm{X}) / N\left(\mathrm{H}_{2}\right)\right]$ can suggest conditions different from those actually in existence, detailed radiative transfer modeling are essential to give more reliable results (Sects. 2 and 4.1). This also underscores the importance of modeling the complete physical, thermal and chemical structure of the envelope when comparing to observations.

3. Water and CO are stable, even at high temperatures, except for destruction by cosmic-ray-driven ionmolecule reactions for $t>10^{5} \mathrm{yrs}$. The ice and gas abundances toward other sources suggest that there may exist a water destruction mechanism at high temperatures $(>500 \mathrm{~K})$. Such a mechanism does not exist in our chemical model, but is worth further exploration (Sects. 4.1 and 4.2).

4. The hydrocarbon and nitrogen chemistry is strongly influenced by temperature, with significant production possible at high $(T \geq 400 \mathrm{~K})$ temperatures. Still it appears that there may exist an as-yet unexplored path to $\mathrm{HCN}$ for $T \geq 600 \mathrm{~K}$ (Sect. 4.2).

5 . The sulphur chemistry displays three significant regimes. In the cool exterior, over $50 \%$ of the sulphur exists in CS. For $100 \leq T(\mathrm{~K})<300, \mathrm{SO}_{2}$ contains a majority of the sulphur. At higher temperatures, atomic sulphur dominates. While consistent with observations, these results employ a low gas-phase sulphur abundance. We cannot identify the principle sulphur reservoir in the cold gas (Sect. 4.3).

6. Carbon dioxide may possibly be destroyed by reactions with molecular hydrogen in impulsive heating events - a reaction which requires further study. The suggestion that $\mathrm{CO}_{2}$ may be destroyed by $\mathrm{H}$ has problems in that it may destroy too much $\mathrm{CH}_{4}$ in the interior and produce too much atomic and molecular oxygen (Sect. 4.4).

7. The cosmic-ray ionization rate in AFGL 2591 is important. It affects the ion abundances as well as the formation of HCN. These processes fix the cosmic ray ionization rate to better than a factor of three. Ions produced by cosmic rays can also effectively destroy $\mathrm{H}_{2} \mathrm{O}$ on timescales of $>10^{5}$ years (Sect. 4.5).

8. We produce simulated column densities of predicted abundant species for comparison with future observations (Table 3). We predict, among other things, abundant atomic oxygen and nitrogen - even though oxygen can be shuttled to water and $\mathrm{O}_{2}$ while nitrogen is at most $1 \%$ atomic with the majority in $\mathrm{N}_{2}$. The temperature variation within the source can have a large affect on oxygen, and hence on the rest of the chemistry (Sect. 4.6).

9. While it is possible for the observed $\mathrm{H}_{2} \mathrm{CO}$ abundance to be reproduced by gas-phase chemistry, the same is not true for $\mathrm{CH}_{3} \mathrm{OH}$. This suggests that some other processes are important for $\mathrm{CH}_{3} \mathrm{OH}$, including grainsurface chemistry and/or the potential of a heating event within the envelope (Sect. 4.7).

10. It is possible to use detailed chemical modeling to constrain the chemical age of AFGL 2591. We find $7 \times 10^{3} \leq t(\mathrm{yrs}) \leq 5 \times 10^{4}$, with a strong preference for $t \sim 3 \times 10^{4}$ years (Sect. 5 ).

11. The agreement of our results with the line data lend significant further confirmation to the physical model proposed by van der Tak et al. (2000), and the chemical structure and evolution proposed here.

Acknowledgements. We are grateful to the anonymous referee for comments and discussions that helped improve the presentation. This work was partially supported under grants from The Research Corporation (SDD), and the Netherlands Organisation for Scientific Research (NWO) through grant 614-41-003 (AB, FvdT), and a NWO bezoekersbeurs. Astrochemistry at Leiden is supported through an NWO Spinoza award (EvD).

\section{References}

Aitken, D. K., Roche, P. F., Smith, C. H., et al. 1988, MNRAS, 230,629

Bally, J., \& Lada, C. J. 1983, ApJ, 265, 824

Bergin, E. A., Langer, W. D., \& Goldsmith, P. F. 1995, ApJ, 441, 222

Beuther, H., Schilke, P., Menten, K., et al. 2002, ApJ, 566, 945

Boonman, A. M. S., et al. 2001, ApJ, 553, L63

Boonman, A. M. S., et al. 2000, in ISO beyond the peaks, ed. A. Salama, M. F. Kessler, K. Leech, \& B. Schulz, ESA-SP 456, 67 [astro-ph/0105249]

Brown, P. D., \& Charnley, S. B. 1990, MNRAS, 244, 432

Carr, J. S., Evans, N. J., Lacy, J. H., \& Zhou, S. 1995, ApJ, 450,667

Charnley, S. B. 1997, ApJ, 481, 396

Charnley, S. B., \& Kaufman, M. J. 2000, ApJ, 529, L111

Choi, M., Evans, N. J., Jaffe, D. T., \& Walker, C. 1994, ApJ, 435,734

Churchwell, E. B. 1993, in ASP Conf. Ser., 35, Massive Stars, Their Lives in the Interstellar Medium, ed. J. P. Cassinelli, \& E. B. Churchwell, ASP, 35

Churchwell, E. B. 1999, in The Physics of Star Formation and Early Stellar Evolution II, ed. C. J. Lada, \& N. D. Kylafis (Kluwer), 515

de Graauw, T., et al. 1996, A\&A, 315, L345 
Doty, S. D., \& Neufeld, D. A. 1997, ApJ, 489, 122

Doty, S. D., van Dishoeck, E. F., van der Tak, F. F. S., et al. 2002, A\&A, submitted

Draine, B. T., Roberge, W. G., \& Dalgarno, A. 1983, ApJ, 264, 485

Egan, M. P., Leung, C. M., \& Spagna, G. R. Jr. 1988, Comput. Phys. Commun., 48, 271

Ehrenfreund, P., Dartois, E., Demyk, K., \& D'Hendecourt, L. 1998, A\&A, 339, L17

Fraser, H. J., Collings, M. P., McCoustra, M. R. S., \& Williams, D. A. 2001, MNRAS, 327, 1165

Garay, G., \& Lizano, S. 1999, PASP, 111, 1049

Gensheimer, P. D., Mauersberger, R., \& Wilson, T. L. 1996, A\&A, 314, 281

Gerakines, P., Whittet, D. C. B., Ehrenfreund, P., et al. 1999, ApJ, 522, 357

Goldsmith, P. F., Melnick, G. J., Bergin, E. A., et al. 2000, ApJ, 539, L123

Graven, W. M., \& Long, F. J. 1954, J. Am. Chem. Soc., 76, 2602

Gwenlan, C., Ruffle, D. P., Viti, S., et al. 2000, A\&A, 354, 1127

Hatchell, J., et al. 1998, A\&A, 338, 713

Hatchell, J., Fuller, G. A., Miller, T. J., Tompson, M. A., \& Macdonald, G. H. 2000, A\&A, 357, 637

Helmich, F. P. 1996, Ph.D. Thesis, Leiden University

Helmich, F., Jansen, D. J., de Graauw, Th., Groesbeck, T. D., \& van Dishoeck, E. F. 1994, A\&A, 283, 626

Helmich, F., van Dishoeck, E. F., Black, J. H., et al. 1996, A\&A, 315, L173

Herbst, E. 1995, in ASSL Vol. 202: The Diffuse Interstellar Bands, 307

Herbst, E., \& Klemperer, W. 1973, ApJ, 185, 505

Hollenbach, D., Johnstone, J., Lizano, S., \& Shu, F. 1994, ApJ, 428,654

Irvine, W. M., Ohishi, M., \& Kaifu, N. 1991, Icarus, 91, 2

Keane, J. V., Boonman, A. M. S., Tielens, A. G. G. M., \& van Dishoeck, E. F. 2001, A\&A, 376, L5

Knez, C., et al. 2002, AAS, 134.10

Kurtz, S., Cesaroni, R., Churchwell, E., Hofner, P., \& Walmsley, M. 2000, in Protostars and Planets IV, ed. V. Manning, A. Boss, \& S. Russel (Univ. of Arizona Press), 299

Lada, C. J., Thronson, H. A. Jr., Smith, H. A., et al. 1984, ApJ, 286, 302

Lahuis, F., \& van Dishoeck, E. F. 2000, A\&A, 355, 699

Leung, C. M., Herbst, E., \& Huebner, W. F. 1984, ApJS, 56, 231

McCall, B. J., Hinkle, K. H., Geballe, T. R., et al. 2002, ApJ, 567,391

McCall, B. J., Geballe, T. R., Hinkle, K. H., \& Oka, T. 1999, ApJ, 522, 338

Melnick, G. A., Ashby, M. L. N., Plume, R., et al. 2000, ApJ, 539, L87
Meyer, D. M., Jura, M., \& Cardelli, J. A. 1998, ApJ, 493, 222

Millar, T. J. 1990, In Molecular Astrophysics: A Volume Honoring Alexander Dalgarno, ed. T. W. Harquist (Cambridge, Cambridge University Press), 114

Millar, T. J. 1993, in Dust and Chemistry in Astronomy, ed. T. J. Millar, \& D. A. Williams (IOP Publishing, Bristol), 249

Millar, T., Farquhar, P., \& Willacy, K. 1997, A\&AS, 121, 193

Millar, T. J., \& Freeman, A. 1984, MNRAS, 207, 405

Minh, Y. C., Irvine, W. M., McGonagle, D., \& Ziurys, L. M. 1990, ApJ, 360, 136

Mitchell, G. F., Curry, C., Maillard, J., \& Allen, M. 1989, ApJ, 341,1020

Neufeld, D. A., Ashby, M. L. N., Bergin, E. A., et al. 2000, ApJ, 539, L111

Neufeld, D. A., Lepp, S., \& Melnick, G. J. 1995, ApJS, 100, 132

Prasad, S. S., \& Huntres, W. T. Jr. 1980, ApJS, 43, 1

Richling, S., \& Yorke, H. W. 1997, A\&A, 327, 317

Rodgers, S. D., \& Charnley, S. B. 2001, ApJ, 546, 324

Smith, C. H., et al. 2000, MNRAS, 312, 327

Snell, R. L., Howe, J. E., Ashby, M. L. N., et al. 2000, ApJ, 539, L101

Stahler, S. W. 1984, ApJ, 281, 209

Talbi, D., \& Herbst, E. 2002, A\&A, in press

Talbi, D., \& Herbst, E. 1998, A\&A, 333, 1007

Tielens, A. G. G. M., Tokunaga, A. T., Geballe, T. R., \& Baas, F. 1991, ApJ, 381, 181

van der Tak, F. F. S. 2002, in Hot Star Workshop III: The Earliest Phases of Massive Star Birth, ed. P. A. Crowther, ASP Conf. Ser., in press

van der Tak, F. F. S., \& van Dishoeck, E. F. 2000, A\&A, 358, L79

van der Tak, F. F. S., et al. 1999, ApJ, 522, 991

van der Tak, F. F. S., et al. 2000, ApJ, 537, 283

van der Tak, F. F. S., van Dishoeck, E. F., \& Caselli, P. 2000, A\&A, 327

van Dishoeck, E. F. 1998, Faraday Discuss., 109, 31

van Dishoeck, E. F., \& Hogerheijde, M. R. 1999, in The Origins of Stars and Planetary Systems, ed. C. J. Lada, \& N. Kylafis (Kluwer), 97

van Dishoeck, E. F., \& van der Tak, F. F. S. 2000, in Astrochemistry: From Molecular Clouds to Planetary Systems, IAU Symposium 197, ed. Y. C. Minh, \& E. F. van Dishoeck, p. 97

van Dishoeck, E. F., et al. 1996, A\&A, 315, L349

Walmsley, C. M., \& Schilke, P. 1992, in Astrochemistry of Cosmic Phenomena, IAU Symposium 150, ed. P. D. Singh (Kluwer), p. 251

Whittet, D. C. B., et al. 1998, ApJ, 498, L159

Xie, T., Allen, M., \& Langer, W. D. 1995, ApJ, 440, 674 